\title{
Estrutura e relação composição florística-ambiente em uma Floresta Estacional Semidecidual, $\mathrm{MG}$
}

Structure and floristic composition-environment relationship in a seasonal semideciduous forest, Minas Gerais state, Brazil

\author{
L. S. Silva ${ }^{1 *}$; C. C. C. Soares ${ }^{1}$; P. A. Oliveira ${ }^{1}$; C. V. Mendonça Filho²; R. T. \\ Botrel $^{3}$; E. L. M. Machado ${ }^{4}$ \\ ${ }^{1}$ Programa de Pós-graduação em Ciência Florestal, Universidade Federal dos Vales do Jequitinhonha e Mucuri \\ (UFVJM), 39100-000, Diamantina, MG, Brasil. \\ ${ }^{2}$ Departamento de Ciências Biológicas da Universidade Federal dos Vales do Jequitinhonha e Mucuri (UFVJM), \\ CEP 39100-000, Diamantina, MG, Brasil. \\ ${ }^{3}$ Centro de Ciências Agrárias/Universidade Federal Rural do Semi-Árido Universidade Federal Rural do Semi-Árido \\ (UFERSA), CEP: 59.625-900, Av. Francisco Mota, 572 - Bairro Costa e Silva, Mossoró RN, Brasil.| \\ ${ }^{4}$ Departamento de Engenharia Florestal, Universidade Federal dos Vales do Jequitinhonha e Mucuri (UFVJM), \\ 39100-000, Diamantina, MG, Brasil.
}

*leosoares.ef@gmail.com

(Recebido em 26 de janeiro de 2021; aceito em 10 de setembro de 2021)

\begin{abstract}
O objetivo desse estudo foi avaliar a estrutura e a diversidade florística em um fragmento de Floresta Estacional Semidecidual, no Parque Nacional das Sempre-vivas, Minas Gerais, com o propósito de identificar se a composição das espécies está relacionada com as variáveis ambientais e espaciais. Foram alocadas 30 parcelas de $400 \mathrm{~m}^{2}(20 \times 20 \mathrm{~m})$, distribuídas de forma sistemática. Em cada parcela foram identificados e mensurados os indivíduos vivos com diâmetro à altura do peito (DAP) $\geq 5,0 \mathrm{~cm}$. Foram também coletadas amostras de solo (profundidade de $0-20 \mathrm{~cm}$ ), para posteriores análises químicas e físicas. Foram calculados os parâmetros fitossociológicos clássicos, índice de diversidade de Shannon (H') e equabilidade de Pielou (J'), distribuição diamétrica e análises multivariadas de redundância (RDA). As análises edáficas mostraram que essa comunidade possui solo de textura argilosa e baixa disponibilidade de macronutrientes. Já as variáveis ambientais (cota média, cálcio, manganês, cobre, fósforo e areia) tiveram correlação significativa com a distribuição das espéceis $(\mathrm{p}<0,05)$, o componente ambiental e espacial explicaram $15 \%$ da distribuição das espécies. A proporção não explicada indica de que outras variáveis não mensuradas nesse estudo prevalecem na estruturação dessa comunidade. Foram encontrados 2187 indivíduos, 51 famílias botânicas, divididos em: grupo I com 1017 indivíduos e 128 espécies, grupo II 794 indivíduos e 132 espécies e grupo III 376 indivíduos e 97 espécies. As espécies Licania hoehne e Ocotea corymbosa possuem os maiores valores de importância. A distribuição diamétrica mostra que a maioria dos indivíduos se concentram nas primeiras classes diamétricas.
\end{abstract}

Palavras-chave: Diversidade florística, fitossociologia, Reserva da Biosfera.

The aim of this study was to evaluate the structure and floristic diversity in a fragment of Semidecidual Seasonal Forest, in the Sempre-Vivas National Park, Minas Gerais, with the purpose of identifying whether the composition of the species is related to environmental and spatial variables. Thirty plots of $400 \mathrm{~m}^{2}(20$ $\mathrm{m} \times 20 \mathrm{~m}$ ) were distributed systematically. In each plot, living individuals with diameter at breast height (DBH) were identified and measured $\geq 5.0 \mathrm{~cm}$. Soil samples (depth of $0-20 \mathrm{~cm}$ ) were also collected for further chemical and physical analyses. Classical phytosociological parameters, Shannon diversity index $\left(\mathrm{H}^{\prime}\right)$ and Pielou equability $\left(\mathrm{J}^{\prime}\right)$, diametric distribution and multivariate redundancy analyses (RDA) were calculated. The edaphytic analyses show that this community has clay soil and low availability of macronutrients. On the other hand, the environmental variables (average quota, calcium, manganese, copper, phosphorus and sand) had a significant correlation with the distribution of species $(\mathrm{p}<0.05)$, the environmental and spatial component explained $15 \%$ of the species distribution. The unexplained proportion indicates that other variables not measured in this study prevail in the structuring of this community. We found 2,187 individuals, 51 botanical families, divided into: group I with 1017 individuals and 128 species, group II 794 individuals and 132 species and group III 376 individuals and 97 species. The species Licania hoehne and Ocotea corymbosa have the highest values of importance. The diametric distribution shows most individuals focus on the first diametric classes. Keywords: Floristic diversity, phytosociology, Biosphere Reserve. 


\section{INTRODUÇÃO}

Em florestas tropicais têm sido propostos mecanismos para tentar explicar a alta diversidade florística, sendo que a maioria desses estudos tem relacionado à diversidade com a heterogeneidade ambiental [1, 2]. A heterogeneidade é um dos fatores que influenciam a composição das espécies [3] e seus efeitos podem estar relacionados a características edáficas, topográficas, disponibilidade de água e luz $[4,5]$.

Além dos filtros ambientais, os fatores intrínsecos dos organismos associados com as características do ambiente, as relações ecológicas, e a pressão antrópica, constituem alguns dos importantes mecanismos reguladores da riqueza e abundância de espécies nas comunidades [6, 7]. Já a competição e os mecanismos de dispersão podem influenciar diretamente na distribuição das espécies vegetais [8]. O espaço também é considerado como um dos fatores ecológicos mais importantes na estruturação e distribuição das espécies [5].

É importante considerar também os eventos estocásticos tais como colonização por acaso, extinção aleatória e deriva ecológica (Teoria Neutra) [9]. Ao longo do tempo, a fragmentação vem provocando mudanças na composição florística e na estrutura das florestas [10]. Isso têm levado à perda de espécies, substituição de especialistas por generalistas e redução das funções ecológicas [11].

Nesse contexto, é importante entender os aspectos relacionados com a composição das espécies e dos mecanismos ecológicos que atuam nos padrões estruturais das comunidades florestais, assim, será possível garantir com mais afinco medidas de conservação de sua diversidade [2]. Por meio dos estudos florísticos e fitossociológicos é possível conhecer a flora dos remanescentes florestais, sendo estes essenciais para o entendimento da distribuição das espécies [12]. Esses estudos fornecem relevantes orientações para o manejo e definição de áreas estratégicas para conservação, principalmente em ambientes fragmentados como as Florestas Estacionais Semideciduais, que são provavelmente um dos ecossistemas mais ameaçados e fragmentados do planeta [13].

Desse modo, o objetivo desse estudo foi avaliar a estrutura e a diversidade florística em um fragmento de Floresta Estacional Semidecidual localizado no Parque Nacional das Sempre-Vivas - PNSV, na Reserva da Biosfera da Serra do Espinhaço, com o propósito de identificar se a distribuição das espécies está relacionada com as variáveis ambientais e espaciais.

\section{MATERIAL E MÉTODOS}

O estudo foi realizado em um fragmento de Floresta Estacional Semidecidual - FESD, localizado no Parque Nacional das Sempre-Vivas - PNSV, na Reserva da Biosfera da Mata Atlântica, no município de Buenópolis, no estado de Minas Gerais (Figura 1).

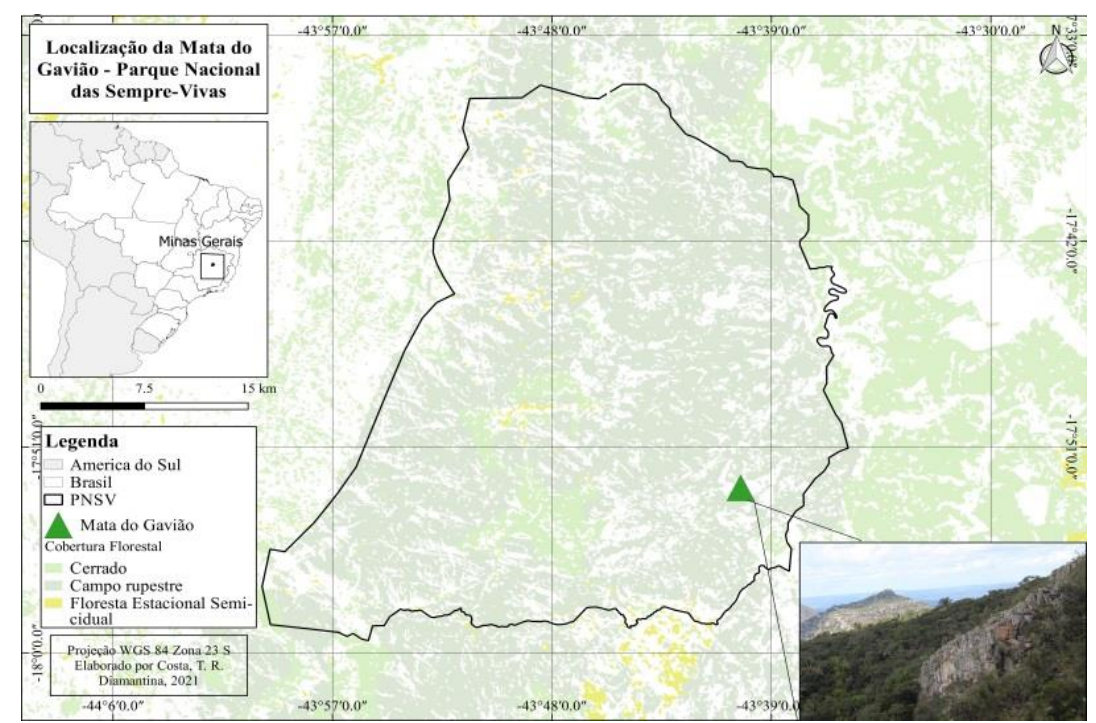

Figura 1. Mata do Gavião, localizado no Parque Nacional das Sempre-Vivas, Minas Gerais, Brasil. 
Esta reserva é conhecida no cenário nacional e internacional, por abrigar nascentes de diferentes bacias hidrográficas: Rio Doce, do Rio Jequitinhonha e do Rio São Francisco [14]. Além de compor uma área ímpar quanto às formações geológicas e florísticas, que apresentam um significativo grau de endemismo de diversas famílias de plantas, abriga grande parte da flora ameaçada de extinção no estado de Minas Gerais $[15,16]$.

O PNSV possui paisagem formada por um mosaico de formações vegetais que se alternam entre afloramentos rochosos sobre diferentes tipos de solo e relevo [17]. Dentre as formações encontradas no PNSV, estão os fragmentos de Floresta Estacional Semidecidual. O fragmento selecionado é denominado Mata do Gavião, e se localiza nas coordenadas $17^{\circ} 52^{\prime} 47.19^{\prime \prime} \mathrm{S}$, $43^{\circ} 40^{\prime} 15.49^{\prime \prime} \mathrm{O}$, com altitude média de $1.168 \mathrm{~m}$.

Segundo a classificação de Köppen o clima é tropical úmido, do tipo $\mathrm{Cwb}$, temperaturas médias anuais de $20^{\circ} \mathrm{C}$ e pluviosidade variando de 1.250 a $1.500 \mathrm{~mm}$ [18]. O fragmento possui aproximadamente 24 hectares. Se encontra na forma de enclave, margeado por fitofisionomias de Cerrado, rochas quartzíticas e por um córrego, sendo dividido em duas porções devido à presença de uma estrada de terra no seu interior. Possui ainda histórico de perturbação antrópica, como retirada seletiva de madeira nos últimos 40 anos, antes da implementação do Parque.

\subsection{Amostragem do componente arbóreo}

A vegetação arbórea foi amostrada em 30 parcelas quadradas de $400 \mathrm{~m}^{2}(20 \times 20 \mathrm{~m})$ cada, totalizando 1,2 ha de área amostral. As parcelas foram alocadas de forma sistemática, distanciadas $80 \mathrm{~m}$ entre si. Em cada parcela foram identificados e mensurados os indivíduos vivos com diâmetro à altura do peito $(\mathrm{DAP}) \geq 5,0 \mathrm{~cm}$. Os indivíduos com troncos múltiplos foram mensurados quando a raiz da soma dos quadrados do DAP foi $\geq 5,0 \mathrm{~cm}$ (diâmetro quadrático).

Os espécimes, quando não identificadas em campo foram coletado material vegetativo e, posteriormente foram encaminhados para o Herbário Dendrológico Jeanine Felfili - HDJF. Foram identificadas por meio de consultas à literatura (http://www.splink.org.br), por especialistas e por comparação com as exsicatas do acervo do HDJF da Universidade Federal dos Vales do Jequitinhonha e Mucuri (UFVJM). A classificação botânica foi realizada por meio do sistema APG IV (2016) [19].

\subsection{Coleta das variáveis ambientais}

Em cada parcela, foram coletadas cinco amostras simples do solo superficial $(0-20 \mathrm{~cm})$, as quais foram homogeneizadas. Posteriormente separou-se uma amostra composta, com cerca de $500 \mathrm{~g}$ de solo por parcela. Em seguida foram realizadas as análises químicas: $\mathrm{pH}$ (água), teor de fósforo $(\mathrm{P})$, teor de potássio $(\mathrm{K})$, teor de cálcio $(\mathrm{Ca})$, teor de magnésio $(\mathrm{Mg})$, teor de alumínio (Al), capacidade de troca de cátions à pH $7(\mathrm{~T})$, saturação por $\mathrm{Al}(\mathrm{m})$, saturação por bases $(\mathrm{V})$ [20], e textural para determinação da porcentagem de areia, silte e argila [21]. Esse procedimento foi realizado no Laboratório de Análises de Solos da Universidade Federal dos Vales do Jequitinhonha e Mucuri - UFVJM, conforme o protocolo da EMBRAPA (2018) [22].

As variáveis topográficas foram obtidas para cada parcela. Para tal, foram coletadas a inclinação entre os vértices e entre as parcelas, com auxílio de um clinômetro tipo Abney (CST17645) e uma trena. Com as coordenadas dos vértices de cada parcela foram obtidas duas variáveis: a) 'cota média', obtida a partir da média das cotas de cada vértice da parcela e b) 'desnível', obtido pela diferença entre as cotas máximas e mínimas [23]. Foram também obtidas a distância entre as parcelas e um córrego que contorna o fragmento.

\subsection{Análises multivariadas}

Análises Canônicas de Redundância (RDA's) foram utilizadas para verificar a fração de explicação das variáveis preditoras (ambientais) sobre os padrões da composição das espécies. Para esse procedimento foram empregadas três matrizes: a primeira matriz com as coordenadas geográficas de cada parcela, a segunda com os dados de presença e ausência das espécies e a terceira composta pelas variáveis ambientais de cada parcela (propriedades químicas, texturais e 
topográficas do solo e distância do córrego). As coordenadas geográficas foram utilizadas para criar variáveis espaciais (MEMs - Moran's Eigenvector Maps) e entender melhor a influência do espaço na distribuição das espécies na comunidade, para esse procedimento foi usado o default do pacote 'vegan'. Foram criados $24 \mathrm{MEMs}$, dentre os quais apenas três foram significativos e selecionados para o modelo pelo método forward selection [24]. A significância dos resultados da RDA foi testada pelo teste de permutação com 999 repetições para o modelo global. Foi realizado o processamento da RDA parcial de todas as variáveis mencionadas anteriormente e posteriormente com as variáveis que se mostraram correlacionadas.

O processamento dos dados da RDA foi realizado no software R (R Development Core Team 2016) [25], usando especificamente os pacotes vegan [26], ade4 [27], packfor [28], spacemakeR [28], venneuler [29] e tripack [30].

\subsection{Estrutura horizontal}

Os parâmetros fitossociológicos da estrutura horizontal foram calculados conforme MuellerDombois e Ellemberg (2002) [31]: densidade relativa (DR), área basal (AB), dominância relativa (DoR), frequência relativa (FR) e valor de importância (VI). A diversidade florística foi determinada pelo índice de diversidade de Shannon ( $\left.\mathrm{H}^{\prime}\right)$ e equabilidade de Pielou (J') [32].

\subsection{Distribuição diamétrica}

A distribuição das classes diamétricas foi realizada por meio de intervalos com amplitudes crescentes, para compensar o forte decréscimo da densidade nas classes de tamanhos maiores, típico da distribuição em exponencial negativo, como adotado em estudos de Botrel et al. (2002) [33]. Divididas da seguinte forma: classe 1 - 5 a $10 \mathrm{~cm}$ de DAP; classe 2 - de 10,1 a $20 \mathrm{~cm}$ de DAP; classe 3 - de 20,1 a $40 \mathrm{~cm}$ de DAP e classe 4 acima de 40,1 $\mathrm{cm}$ de DAP.

\section{RESULTADOS E DISCUSSÃO}

As análises indicam solo argiloso com baixa disponibilidade de macronutrientes, sendo classificado como distrófico [34] $\left(\mathrm{P}=1,15 \mathrm{mg} / \mathrm{dm}^{3}, \mathrm{Mg}^{2+}=0,07 \mathrm{cmolc} / \mathrm{dm}^{3}, \mathrm{Ca}^{2+}=0,23\right.$ cmolc/ $\left./ \mathrm{dm}^{3}, \mathrm{~V}=2,85 \%\right)$ e ácido $(\mathrm{pH}=3,72)$ segundo o critério estabelecido pela Embrapa (2006) [35]. Os teores de $\mathrm{Al}^{3+}$, acidez potencial $(\mathrm{H}+\mathrm{Al})$ e saturação por alumínio $(\mathrm{m})$ indicam excesso de íons de alumínio. Os baixos valores da soma de bases (SB) e de saturação (V) encontrados revelam os altos níveis de acidez e baixa disponibilidade de cátions básicos (Tabela suplementar (<http://dx.doi.org/10.6084/m9.figshare.16601738>). Os elementos essenciais P, K, Ca e Mg estão em níveis considerados como baixos a muito baixos pela literatura [34].

Os gráficos da RDA mostram que três variáveis espaciais apresentaram influência na estruturação com o componente arbóreo (MEM1, MEM6 e MEM17) (Figura 2A). Na Figura 2B, é possível notar o padrão de distribuição da comunidade em função das variáveis ambientais e espaciais, em sua maioria ocupam setores distintos. 

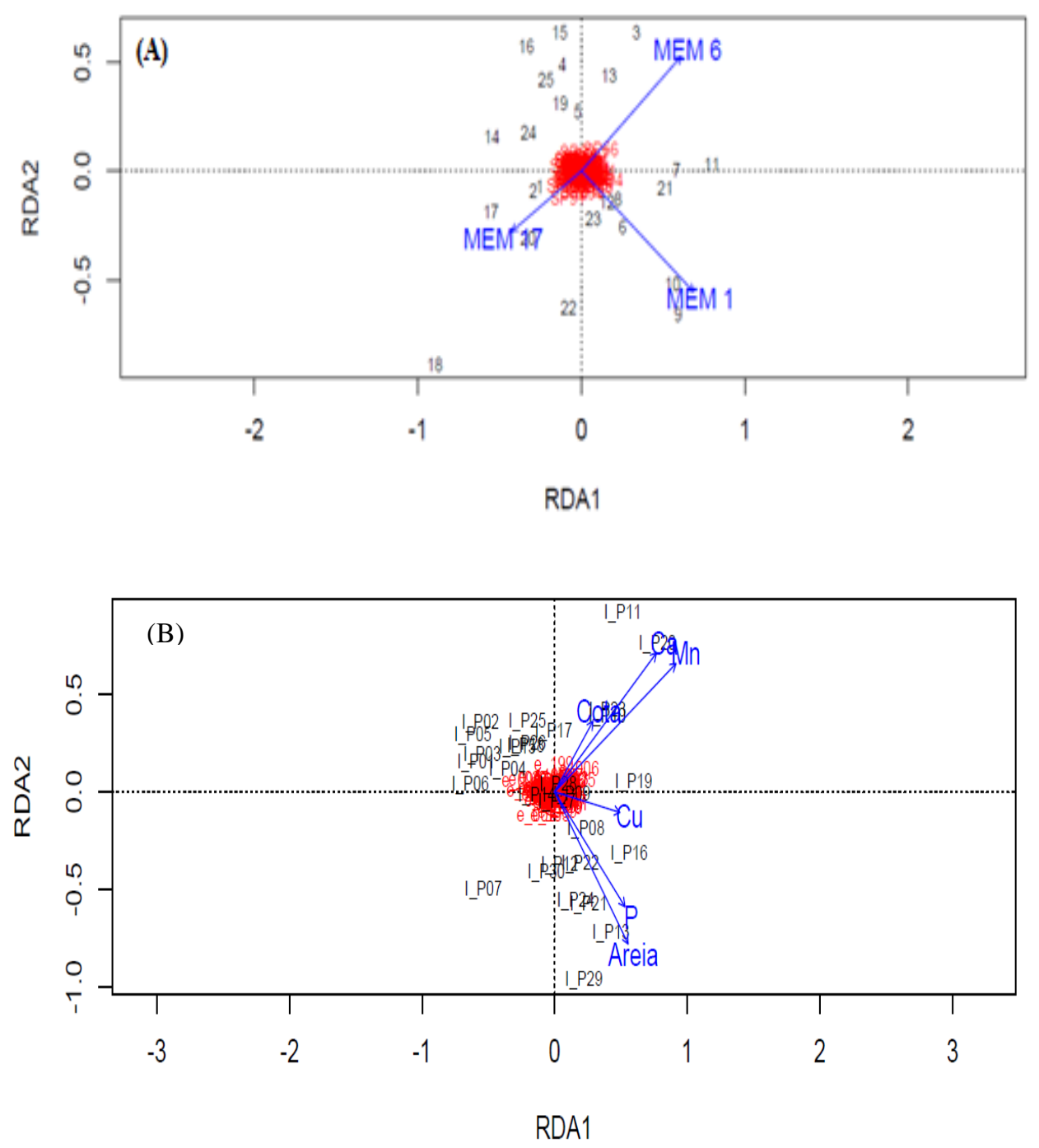

Figura 2. Análises de Redundância (RDAs) em função de variáveis espaciais (A) e ambientais (B), para 30 parcelas amostradas no fragmento de Floresta Estacional Semidecidual Mata do Gavião no Parque Nacional das Sempre-Vivas, Reserva da Biosfera do Espinhaço - MG. Os pontos vermelhos são as espécies.

As espécies se concentraram na parte central do diagrama, ou seja, não há separação ao longo do eixo em virtude das variáveis ambientais utilizadas. Segundo Souza et al. (2017) [36] quando as espécies se concentram no centro do diagrama significa que elas têm maior plasticidade fenotípica em relação às variáveis ambientais.

Já as variáveis ambientais cota média, cálcio, manganês, cobre, fósforo e areia tiveram correlação significativa com a composição de espécies $(\mathrm{p}<0,05)$, ou seja, em parte, o ambiente determina a distribuição das espécies. As figuras $2 \mathrm{~B}$ e 3 , mostram a formação de três grupos de parcelas sendo o grupo I formado pelas parcelas que não possuem correlação com as variáveis ambientais (P01, P02, P03, P04, P05, P06, P07, P15, P17, P18 e P25 e 26); o grupo II formado pelas parcelas que se correlacionaram com as variáveis cobre, fósforo e areia (P08, P09, P12, P13, P14, P16, P21, P22, P24, P27, P29 e P30); e o grupo III, formado pelas unidades amostrais que tiveram correlação com as variáveis ambientais cota média, cálcio e manganês (P10, P11, P19, P20, P23 e P28). 

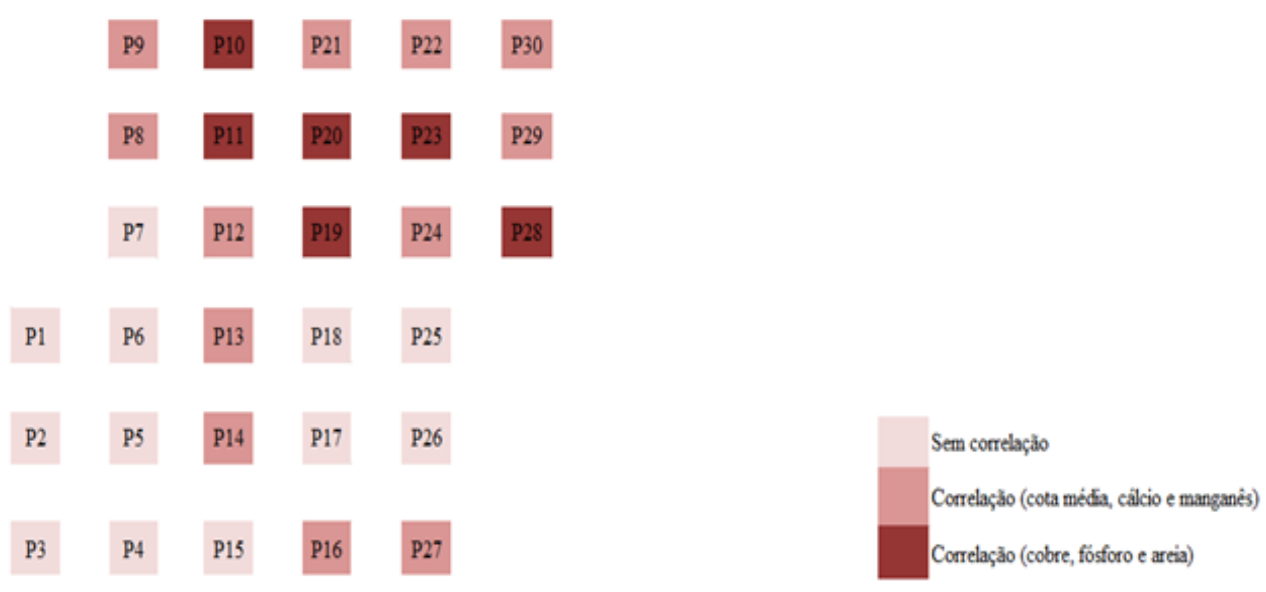

Figura 3. Ilustração das parcelas que apresentaram ou não correlação com as variáveis ambientais na Mata do Gavião, Parque Nacional das Sempre-Vivas, Minas Gerais.

Os grupos II e III são as parcelas que se encontram em altitude mais baixa em relação às parcelas do grupo I. As maiores concentrações dos elementos foram encontadas nas parcelas dos grupos II e III, o que reflete a existência de interação entre espécies e fatores ambientais e topográficos.

Foi observado que essas variáveis explicaram $14 \%(\mathrm{~F}=1,632, \mathrm{p}=0,005)$ do valor total, bem superior ao espaço que explicou $1 \%(\mathrm{~F}=1,299, p=0,021)$. A variação explicada conjuntamente pelas duas frações foi baixa (3\%). Os resultados reforçam a importância dos fatores ambientais, pois essas variáveis explicaram sozinhas a maior parte da estruturação (14\%) (Figura 4).

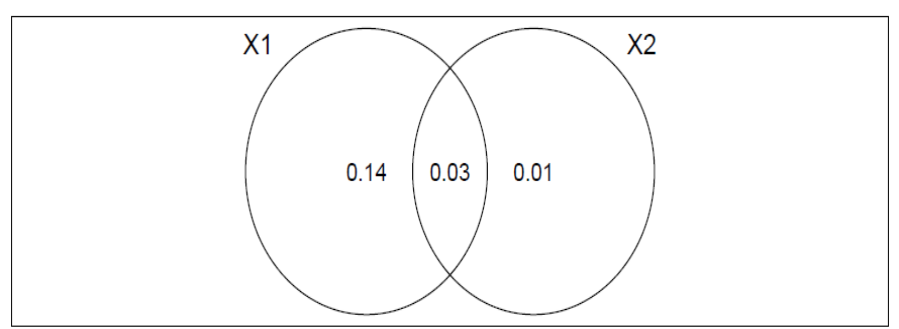

Figura 4. Diagrama de Venn da partição da variação da organização florístico-estrutural do componente arbóreo de um fragmento de Floresta Estacional Semidecidual, Mata do Gavião Parque Nacional das Sempre-Vivas - MG. [x1] Variação explicada puramente pelas variáveis ambientais; [x2] variação explicada puramente pelas variáveis espaciais e [interseção xl/x2] variação explicada de forma compartilhada pelas variáveis ambientais e espaciais.

O valor da partição da variância (82\%) não foi explicado pelos preditores ambientais e espaciais selecionados (Figura 4). Segundo Carvalho et al. (2007) [37], isso não compromete a relação espécie-ambiente das variáveis testadas, ou seja, existe outras variáveis não mensuradas nesse estudo que devem explicar em maior parte essa relação. Segundo Maçaneiro et al. (2016) [3]; Loebens et al. (2018) [38], a porcentagem não explicada é comum em estudos que avaliam o poder preditor das variáveis ambientais sobre a heterogeneidade florística.

Maracahipes-Santos et al. (2017) [39] e Dias (2019) [40] afirmaram que em fitofisionomias de Cerrado e Mata Atlântica é comum o alto percentual não explicado para a variação florística. Portanto, isso reforça a idéia de que outras variáveis não determinadas por essas análises atuam nos padrões florísticos e estruturais e que estes são inerentes da própria comunidade, independentemente das condições ambientais [38].

Neste sentido, os resultados sugerem a importância de fatores de ordem endógena (como dispersão e competição intra e interespecífica) na estruturação e formação de manchas de indivíduos coespecíficos [37, 41], tendo sua distribuição determinada pelos fatores bióticos [42, 43], ou eventos estocásticos como sugerido pela Teoria Neutra, ou seja, as diferenças florísticas 
são criadas de forma aleatória, porém limitadas pela dispersão e por eventos vicariantes [9]. Bem como as variáveis ambientais não incluídas na análise (como microclima, outros preditores de solo, etc) também podem ter contribuído para a porção não explicada pelo ambiente e espaço.

Em relação aos grupos de parcelas foram contabilizados um total de 2187 indivíduos e 51 famílias botânicas e 206 espécies: grupo I com 1017 indivíduos e 128 espécies; o grupo II com 794 indivíduos e 132 espécies; grupo III com 376 indivíduos e 97 espécies (Tabela 1).

Tabela 1. Valores dos parâmetros estimados para cada grupo no fragmento de Floresta Estacional Semidecidual, Mata do Gavião, Parque Nacional das Sempre-Vivas, MG.

\begin{tabular}{lllllllllll}
\hline Grupos & $\mathrm{NP}$ & $\mathrm{Ni}$ & N.Esp. & NspE & H' & J & DA & FA & DoA & AB \\
\hline I & 12 & 1017 & 128 & 44 & 3,8 & 0,7 & 847,50 & 1216,66 & 11,83 & 14,20 \\
II & 12 & 794 & 132 & 36 & 4,0 & 0,8 & 661,66 & 1086,66 & 11,93 & 14,32 \\
III & 6 & 376 & 98 & 19 & 4,0 & 0,8 & 313,33 & 560,00 & 5,81 & 6,96 \\
Total & 30 & 2.187 & 206 & - & 3,9 & 0,8 & $1.822,49$ & $2.863,32$ & 29,57 & 35,48 \\
\hline
\end{tabular}

Em que: $\mathrm{NP}=$ número de parcelas; $\mathrm{Ni}=$ número de indivíduos; $\mathrm{N}$. Esp. = número de espécies; $\mathrm{NspE}=$ número de espécies exclusivas; $\mathrm{H}^{\prime}$ = índice de diversidade de Shannon (nats/indivíduos); J' = equabilidade de Pielou; DA = densidade absoluta (ind./ha); FA = frequência absoluta; DoA = dominância absoluta $\left(\mathrm{m}^{2} / \mathrm{ha}\right)$ e $\mathrm{AB}=$ área basal $\left(\mathrm{m}^{2}\right)$.

Os valores de diversidade foram iguais aos encontrados em alguns estudos em Florestas Semideciduais do estado de Minas Gerais, que variam entre 3,2 e 4,05 [44] em Capelinha, MG, e [2] em um fragmento localizado no Parque Estadual do Biribiri.

A elevada riqueza de espécies encontrada nessa comunidade pode ser considerada acima do padrão normalmente encontrado na literatura [2, 44, 45], esse fragmento possui influência florística com diferentes fitofisionomias de Cerrado, isso provavelmente pode ter contribuído para a elevada riqueza de espécies. Esse fragmento já foi alvo de retirada seletiva de madeira antes da criação do parque, desde então, não foi registrado distúrbios dessa natureza, isso mostra a importância do parque para conservação das espécies.

Os valores da equabilidade (J') revelam uma distribuição individual uniforme e baixa dominância ecológica entre as espécies. O valor de $\left(\mathrm{J}^{\prime}\right)$ mostra que $80 \%$ da diversidade hipotética máxima $\left(\mathrm{H}^{\prime}\right)$ foi obtida, indicando uma possível disponibilidade intermediária de recursos do solo [46].

Conforme a Tabela 1, as parcelas que não se correlacionaram com as variáveis ambientais, o grupo I, tiveram maior número de indivíduos, espécies exclusivas e densidade absoluta, ou seja, as populações são mais adensadas. As unidades amostrais que se correlacionaram, grupos II e III, foram observados os maiores valores de diversidade florística e equabilidade. No grupo III os valores de densidade, frequência e dominância absolutos são mais de 50\% menores do que os grupos I e II.

Considerando os três grupos, o valor da área basal total é considerado alto se comparado a outros estudos em formações de florestas estacionais que ocorrem na Reserva da Biosfera da Serra do Espinhaço Meridional [2, 23, 47].

As famílias que apresentaram a maior riqueza de espécies foram: Myrtaceae (34), Fabaceae (27), Lauraceae (20), Euphorbiaceae (10), Melastomataceae (8) e Rubiaceae (7). Essas famílias representam 51,4\% da flora amostrada (Tabela 2) e possuem grande destaque nos estudos em Floresta Estacional Semidecidual no estado de Minas Gerais [2, 48].

As espécies das famílias Myrtaceae e Lauraceae a qual se destacaram nesse estudo são muito importantes para a dinâmica e restauração ecológica, pois contribuem como fonte de alimentos para fauna local e, consequentemente, a entrada de propágulos de diferentes plantas [49]. Já as espécies da família Fabaceae ajudam na fixação de nitrogênio no solo e incorporação de matéria orgânica. Estas características ajudam a promover alterações microambientais permitindo, assim, o estabelecimento de espécies, principalmente em condições de solos de baixa fertilidade, e possuem plasticidade ambiental [50].

No grupo I, Licania hoehnei, Ocotea corymbosa, Cabralea canjerana, Amaioua guianensis, Mabea piriri, Thyrsodium spruceanum, Hirtella floribunda, morfotipo, Ocotea aciphylla e Myrcia splendens contribuíram com 53,9\% da densidade total de indivíduos. São essas mesmas 
espécies que possuem as maiores frequências, área basal, dominância relativa e valor de importância. Destaque para Licania hoehnei que mesmo tendo menos indivíduos que Ocotea corymbosa, possui mais que o dobro da área basal e dominância relativa (Tabela 2). Os gêneros que apresentaram o maior número de espécies no grupo I foram Myrcia (11) e Aspidosperma (6) espécies.

Tabela 2. Parâmetros florísticos e fitossociológicos para o fragmento de Floresta Estacional Semidecidual no Parque Nacional das Sempre-Vivas-PNSV, MG. As espécies estão organizadas em ordem decrescente de valor de importância.

\begin{tabular}{|c|c|c|c|c|c|c|c|}
\hline Famílias & Espécies & $\mathbf{N i}$ & DR & FR & $\mathbf{A B}$ & DoR & VI\% \\
\hline \multicolumn{8}{|l|}{ Grupo I } \\
\hline Chrysobalanaceae & Licania hoehnei Pilg. & 105 & 10,32 & 3,01 & 3,3905 & 23,88 & 12,40 \\
\hline Lauraceae & Ocotea corymbosa (Meisn.) Mez & 113 & 11,11 & 3,01 & 1,4700 & 10,35 & 8,16 \\
\hline Meliaceae & Cabralea canjerana (Vell.) Mart. & 50 & 4,92 & 3,29 & 0,7852 & 5,53 & 4,58 \\
\hline Rubiaceae & Amaioua guianensis Aubl. & 66 & 6,49 & 3,29 & 0,4533 & 3,19 & 4,32 \\
\hline Euphorbiaceae & Mabea piriri Aubl. & 70 & 6,88 & 2,47 & 0,2891 & 2,04 & 3,79 \\
\hline Anacardiaceae & Thyrsodium spruceanum Benth. & 30 & 2,95 & 3,01 & 0,6298 & 4,44 & 3,47 \\
\hline \multirow[t]{2}{*}{ Chrysobalanaceae } & Hirtella floribunda Cham. \& Schltdl. & 36 & 3,54 & 2,74 & 0,3331 & 2,35 & 2,88 \\
\hline & Morfotipo & 20 & 1,97 & 2,47 & 0,5171 & 3,64 & 2,69 \\
\hline Lauraceae & Ocotea aciphylla (Nees \& Mart.) Mez & 23 & 2,26 & 2,74 & 0,3989 & 2,81 & 2,60 \\
\hline Myrtaceae & Myrcia splendens (Sw.) DC. & 27 & 2,65 & 2,19 & 0,2127 & 1,50 & 2,11 \\
\hline Lauraceae & Ocotea spixiana $(\mathrm{Nees}) \mathrm{Mez}$ & 23 & 2,26 & 2,19 & 0,2457 & 1,73 & 2,06 \\
\hline Euphorbiaceae & Maprounea guianensis Aubl. & 20 & 1,97 & 2,47 & 0,1260 & 0,89 & 1,77 \\
\hline Euphorbiaceae & Mabea glaziovii Pax \& K.Hoffm. & 26 & 2,56 & 1,10 & 0,1480 & 1,04 & 1,56 \\
\hline Lauraceae & Cryptocarya aschersoniana $\mathrm{Mez}$ & 11 & 1,08 & 1,92 & 0,2362 & 1,66 & 1,55 \\
\hline Fabaceae & Diplotropis ferruginea Benth. & 16 & 1,57 & 1,64 & 0,1432 & 1,01 & 1,41 \\
\hline Rosaceae & Prunus myrtifolia (L.) Urb. & 14 & 1,38 & 1,37 & 0,1778 & 1,25 & 1,33 \\
\hline Lauraceae & Ocotea $\mathrm{sp}$ & 11 & 1,08 & 1,37 & 0,2088 & 1,47 & 1,31 \\
\hline Sapotaceae & Pouteria ramiflora (Mart.) Radlk. & 16 & 1,57 & 1,37 & 0,1177 & 0,83 & 1,26 \\
\hline Lauraceae & Ocotea dispersa (Nees \& Mart.) Mez & 14 & 1,38 & 1,10 & 0,1821 & 1,28 & 1,25 \\
\hline Annonaceae & Xylopia brasiliensis Spreng. & 13 & 1,28 & 1,92 & 0,0678 & 0,48 & 1,22 \\
\hline Lauraceae & Nectandra grandiflora Nees & 13 & 1,28 & 1,64 & 0,0971 & 0,68 & 1,20 \\
\hline Euphorbiaceae & Aparisthmium cordatum (A.Juss.) Baill. & 13 & 1,28 & 1,37 & 0,1177 & 0,83 & 1,16 \\
\hline Fabaceae & Inga schinifolia Benth. & 10 & 0,98 & 1,92 & 0,0785 & 0,55 & 1,15 \\
\hline Annonaceae & Xylopia frutescens Aubl. & 11 & 1,08 & 1,64 & 0,0639 & 0,45 & 1,06 \\
\hline Sapotaceae & Chrysophyllum marginatum (Hook. \& Arn.) Radlk. & 9 & 0,88 & 1,37 & 0,0922 & 0,65 & 0,97 \\
\hline \multirow[t]{2}{*}{ Chrysobalanaceae } & Licania octandra (Hoffmanns. ex Roem. \& Schult.) & & & & & & \\
\hline & Kuntze & 16 & 1,57 & 0,82 & 0,0683 & 0,48 & 0,96 \\
\hline Myrt & Myrcia mischophylla Kiaersk. & 8 & 0,79 & 1,10 & 0,1366 & 0,96 & 0,95 \\
\hline Hypericaceae & Vismia brasiliensis Choisy & 4 & 0,39 & 0,82 & 0,2305 & 1,62 & 0,95 \\
\hline Apocynaceae & Aspidosperma cylindrocarpon Müll.Arg. & 3 & 0,29 & 0,55 & 0,2825 & 1,99 & 0,94 \\
\hline Malpiguiaceae & Byrsonima stannardii W.R.Anderson & 5 & 0,49 & 0,82 & 0,2107 & 1,48 & 0,93 \\
\hline Euphorbiaceae & Alchornea triplinervia (Spreng.) Müll.Arg. & 10 & 0,98 & 0,82 & 0,1286 & 0,91 & 0,90 \\
\hline Euphorbiaceae & Alchornea glandulosa Poepp. \& Endl. & 10 & 0,98 & 1,10 & 0,0880 & 0,62 & 0,90 \\
\hline Myrtaceae & Eugenia florida DC. & 9 & 0,88 & 1,37 & 0,0602 & 0,42 & 0,89 \\
\hline \multirow[t]{2}{*}{ Sapotaceae } & Chrysophyllum gonocarpum (Mart. \& Eichler ex Miq.) & & & & & & \\
\hline & Engl. & 8 & 0,79 & 0,55 & 0,1691 & 1,19 & 0,84 \\
\hline Myrtaceae & Myrcia multiflora (Lam.) DC. & 4 & 0,39 & 0,82 & 0,1670 & 1,18 & 0,80 \\
\hline Theaceae & Laplacea Kunth & 8 & 0,79 & 0,82 & 0,1064 & 0,75 & 0,79 \\
\hline Apocynaceae & Aspidosperma polyneuron Müll.Arg. & 8 & 0,79 & 0,82 & 0,0959 & 0,68 & 0,76 \\
\hline Myrtaceae & Psidium rufum Mart. ex DC. & 3 & 0,29 & 0,55 & 0,1647 & 1,16 & 0,67 \\
\hline Myrtaceae & Myrcia amazonica DC. & 6 & 0,59 & 1,10 & 0,0271 & 0,19 & 0,63 \\
\hline & Siphoneugena densiflora O.Berg & 5 & 0,49 & 1,10 & 0,0319 & 0,22 & 0,60 \\
\hline \multirow[t]{2}{*}{ Fabaceae } & Tachigali friburgensis (Harms) L.G.Silv & & & & & & \\
\hline & H.C.Lima & 4 & 0,39 & 1,10 & 0,0208 & 0,15 & 0,55 \\
\hline Aqui & Ilex brevicuspis Reissek & 4 & 0,39 & 1,10 & 0,0149 & 0,10 & 0,53 \\
\hline Lauraceae & Aniba firmula (Nees \& Mart.) Mez & 1 & 0,10 & 0,27 & 0,1680 & 1,18 & 0,52 \\
\hline Myrtaceae & Myrcia coelosepala Kiaersk & 4 & 0,39 & 0,82 & 0,0429 & 0,30 & 0,51 \\
\hline Annonaceae & Duguetia lanceolata A.St.-Hil. & 3 & 0,29 & 0,55 & 0,0877 & 0,62 & 0,49 \\
\hline Moraceae & Brosimum glaucum Taub & 4 & 0,39 & 0,55 & 0,0703 & 0,49 & 0,48 \\
\hline
\end{tabular}




\begin{tabular}{|c|c|c|c|c|c|c|c|}
\hline Famílias & Espécies & $\mathbf{N i}$ & DR & FR & $\mathbf{A B}$ & DoR & VI\% \\
\hline Peraceae & Pera glabrata (Schott) Baill. & 3 & 0,29 & 0,82 & 0,0395 & 0,28 & 0,46 \\
\hline Combretaceae & Buchenavia tomentosa Eichler & 3 & 0,29 & 0,82 & 0,0240 & 0,17 & 0,43 \\
\hline Euphorbiaceae & Mabea fistulifera Mart. & 7 & 0,69 & 0,27 & 0,0457 & 0,32 & 0,43 \\
\hline Sapindaceae & Simarouba amara Aubl. & 3 & 0,29 & 0,82 & 0,0203 & 0,14 & 0,42 \\
\hline Fabaceae & Hymenolobium janeirense Kuhlm. & 3 & 0,29 & 0,82 & 0,0184 & 0,13 & 0,42 \\
\hline Primulaceae & Cybianthus sp. & 4 & 0,39 & 0,55 & 0,0385 & 0,27 & 0,40 \\
\hline Lauraceae & Ocotea glaziovii Mez & 3 & 0,29 & 0,82 & 0,0131 & 0,09 & 0,40 \\
\hline Myrtaceae & Myrcia obovata (O.Berg) Nied. & 3 & 0,29 & 0,82 & 0,0079 & 0,06 & 0,39 \\
\hline \multirow[t]{2}{*}{ Myrtaceae } & Myrciaria pilosa Sobral \& Couto & 3 & 0,29 & 0,55 & 0,0395 & 0,28 & 0,37 \\
\hline & Morfotipo 1 & 3 & 0,29 & 0,27 & 0,0730 & 0,51 & 0,36 \\
\hline Arecaceae & Attalea oleifera Barb.Rodr. & 1 & 0,10 & 0,27 & 0,0928 & 0,65 & 0,34 \\
\hline Primulaceae & Myrsine coriacea (Sw.) R.Br. ex Roem. \& Schult. & 3 & 0,29 & 0,55 & 0,0157 & 0,11 & 0,32 \\
\hline Elaeocarpaceae & Sloanea monosperma Vell. & 2 & 0,20 & 0,55 & 0,0296 & 0,21 & 0,32 \\
\hline Euphorbiaceae & Actinostemon verticillatus (Klotzsch) Baill. & 3 & 0,29 & 0,55 & 0,0135 & 0,10 & 0,31 \\
\hline Fabaceae & Copaifera langsdorffii Desf. & 2 & 0,20 & 0,55 & 0,0226 & 0,16 & 0,30 \\
\hline Lauraceae & Endlicheria paniculata (Spreng.) J.F.Macbr. & 2 & 0,20 & 0,55 & 0,0161 & 0,11 & 0,29 \\
\hline Apocynaceae & Aspidosperma illustre (Vell.) Kuhlm. \& Pirajá & 2 & 0,20 & 0,55 & 0,0155 & 0,11 & 0,28 \\
\hline Fabaceae & Dalbergia brasiliensis Vogel & 2 & 0,20 & 0,55 & 0,0121 & 0,09 & 0,28 \\
\hline Rubiaceae & Psychotria sessilis Vell. & 2 & 0,20 & 0,55 & 0,0108 & 0,08 & 0,27 \\
\hline Proteaceae & Roupala montana Aubl. & 2 & 0,20 & 0,55 & 0,0087 & 0,06 & 0,27 \\
\hline Melastomataceae & Miconia tristis Spring & 2 & 0,20 & 0,55 & 0,0086 & 0,06 & 0,27 \\
\hline Primulaceae & nbellata Mart. & 2 & 0,20 & 0,55 & 0,0080 & 0,06 & 0,27 \\
\hline Sapindaceae & laeagnoides Radlk. & 2 & 0,20 & 0,55 & 0,0076 & 0,05 & 0,27 \\
\hline Melastomataceae & Miconia cuspidata Naudin & 2 & 0,20 & 0,55 & 0,0059 & 0,04 & 0,26 \\
\hline Rubiaceae & Amaioua intermedia $\mathrm{M}$ & 4 & 0,39 & 0,27 & 0,0120 & 0,08 & 0,25 \\
\hline Myrtaceae & Campomanesia guaviroba (DC.) Kiaersk. & 1 & 0,10 & 0,27 & 0,0527 & 0,37 & 0,25 \\
\hline Myrtaceae & Eugenia capparidifolia DC. & 1 & 0,10 & 0,27 & 0,0430 & 0,30 & 0,23 \\
\hline Myrtaceae & Eugenia cerasiflora Miq. & 1 & 0,10 & 0,27 & 0,0411 & 0,29 & 0,22 \\
\hline Malvaceae & $a$ Mart. \& Zucc. & 1 & 0,10 & 0,27 & 0,0405 & 0,28 & 0,22 \\
\hline Apocynaceae & Aspidosp & 1 & 0,10 & 0,27 & 0,0368 & 0,26 & 0,21 \\
\hline Anacardiaceae & Tapirira obtusa (Benth.) J.D.Mitch. & 1 & 0,10 & 0,27 & 0,0324 & 0,23 & 0,20 \\
\hline Annonaceae & Duguetia chrysocarpa Maas & 1 & 0,10 & 0,27 & 0,0300 & 0,21 & 0,19 \\
\hline Borag & Cord & 1 & 0,10 & 0,27 & 0,0271 & 0,19 & 0,19 \\
\hline Malphiguiaceae & ophylla Kunth & 2 & 0,20 & 0,27 & 0,0113 & 0,08 & 0,18 \\
\hline Anacardiaceae & Schinus terebinthifolia Raddi & 1 & 0,10 & 0,27 & 0,0246 & 0,17 & 0,18 \\
\hline Vochy & Quale & 1 & 0,10 & 0,27 & 0,0 & 0,17 & 0,18 \\
\hline Theaceae & nentosa (Mart.) & 2 & 0,20 & 0,27 & 0,0091 & 0,06 & 0,18 \\
\hline Apocynaceae & Himatanthus lancifolius (Müll. Arg.) Woodsc & 2 & 0,20 & 0,27 & 0,0083 & 0,06 & 0,18 \\
\hline Lauraceae & Cinnaтотит triplinerve (Ruiz \& Pav.) Kosterm. & 2 & 0,20 & 0,27 & 0,0068 & 0,05 & 0,17 \\
\hline Annonaceae & Guatteria australis A.St.-Hil. & 2 & 0,20 & 0,27 & 0,0063 & 0,04 & 0,17 \\
\hline \multirow[t]{2}{*}{ Celastraceae } & rassifolia (Mart. ex Schult.) G.Don & 1 & 0,10 & 0,27 & 0,0155 & 0,11 & 0,16 \\
\hline & Morfotipo 4 & 1 & 0,10 & 0,27 & 0,0147 & 0,10 & 0,16 \\
\hline Och & Ouratea cast & 1 & 0,10 & 0,27 & 0,0141 & 0,10 & 0,16 \\
\hline Fabac & Tachigali $r$ & 1 & 0,10 & 0,27 & 0,0138 & 0,10 & 0,16 \\
\hline Myrtaceae & Myrcia florida Lem. & 1 & 0,10 & 0,27 & 0,0134 & 0,09 & 0,16 \\
\hline Celastraceae & Maytenus boaria Molina & 1 & 0,10 & 0,27 & 0,0114 & 0,08 & 0,15 \\
\hline Myrtac & 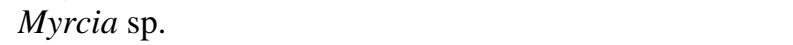 & 1 & 0,10 & 0,27 & 0,0093 & 0,07 & 0,15 \\
\hline Fabaceae & $S$ & 1 & 0,10 & 0,27 & 0,0084 & 0,06 & 0,14 \\
\hline Annonaceae & Annona dolabripetala Raddi & 1 & 0,10 & 0,27 & 0,0080 & 0,06 & 0,14 \\
\hline Rubiaceae & Coutarea hexandra (Jacq.) K.Sch & 1 & 0,10 & 0,27 & 0,0077 & 0,05 & 0,14 \\
\hline Sapotaceae & Chrysophyllum inornatum Mart. & 1 & 0,10 & 0,27 & 0,0076 & 0,05 & 0,14 \\
\hline \multirow[t]{2}{*}{ Fabaceae } & Lonchocarnus cultratus (Vell) A & & & & & & \\
\hline & H.C.Lima & 1 & 0,10 & 0,27 & 0,0075 & 0,05 & 0,14 \\
\hline & Nectandral & 1 & 0,10 & 0,27 & 0,0075 & 0,05 & 0,14 \\
\hline & Calyptranthes & 1 & 0,10 & 0,27 & 0,0070 & 0,05 & 0,14 \\
\hline Myrt: & Euge & 1 & 0,10 & 0,27 & 0,0069 & 0,05 & 0,14 \\
\hline Apocynaceae & Aspidosperma olivaceum Müll.Arg. & 1 & 0,10 & 0,27 & 0,0067 & 0,05 & 0,14 \\
\hline Fabaceae & Libidibia ferrea var. leiostachya (Benth.) L.P.Queiroz & 1 & 0,10 & 0,27 & 0,0063 & 0,04 & 0,14 \\
\hline Myrtaceae & Myrcia guianensis (Aubl.) DC. & 1 & 0,10 & 0,27 & 0,0063 & 0,04 & 0,14 \\
\hline Fabaceae & Tachigali paniculata Aubl. & 1 & 0,10 & 0,27 & 0,0056 & 0,04 & 0,14 \\
\hline Polygonaceae & Coccoloba warmingii Meisn. & 1 & 0,10 & 0,27 & 0,0052 & 0,04 & 0,14 \\
\hline
\end{tabular}




\begin{tabular}{|c|c|c|c|c|c|c|c|}
\hline Famílias & Espécies & Ni & DR & FR & $\mathbf{A B}$ & DoR & VI\% \\
\hline Fabaceae & Abarema villosa Iganci \& M.P.Morim & 1 & 0,10 & 0,27 & 0,0044 & 0,03 & 0,13 \\
\hline \multirow[t]{2}{*}{ Myrtaceae } & Eugenia sp1. & 1 & 0,10 & 0,27 & 0,0043 & 0,03 & 0,13 \\
\hline & Morfotipo 3 & 1 & 0,10 & 0,27 & 0,0041 & 0,03 & 0,13 \\
\hline Lythraceae & Lafoensia vandelliana Cham. \& Schltdl. & 1 & 0,10 & 0,27 & 0,0040 & 0,03 & 0,13 \\
\hline Myrtaceae & Myrcia fenzliana O.Berg & 1 & 0,10 & 0,27 & 0,0037 & 0,03 & 0,13 \\
\hline Lauraceae & Persea rufotomentosa Nees \& Mart. & 1 & 0,10 & 0,27 & 0,0035 & 0,02 & 0,13 \\
\hline Sapindaceae & Cupania ludowigii Somner \& Ferrucci & 1 & 0,10 & 0,27 & 0,0034 & 0,02 & 0,13 \\
\hline Melastomataceae & Miconia ligustroides (DC.) Naudin & 1 & 0,10 & 0,27 & 0,0034 & 0,02 & 0,13 \\
\hline Apocynaceae & Aspidosperma ramiflorum Müll.Arg. & 1 & 0,10 & 0,27 & 0,0033 & 0,02 & 0,13 \\
\hline Lauraceae & Ocotea odorifera (Vell.) Rohwer & 1 & 0,10 & 0,27 & 0,0033 & 0,02 & 0,13 \\
\hline Proteaceae & Roupala brasiliensis Klotzsch & 1 & 0,10 & 0,27 & 0,0031 & 0,02 & 0,13 \\
\hline Melastomataceae & Mouriri glazioviana Cogn. & 1 & 0,10 & 0,27 & 0,0030 & 0,02 & 0,13 \\
\hline Sapindaceae & Cupania vernalis Cambess. & 1 & 0,10 & 0,27 & 0,0030 & 0,02 & 0,13 \\
\hline Calophyllaceae & Kielmeyera sp. & 1 & 0,10 & 0,27 & 0,0029 & 0,02 & 0,13 \\
\hline Sapindaceae & Cupania rubiginosa (Poir.) Radlk. & 1 & 0,10 & 0,27 & 0,0028 & 0,02 & 0,13 \\
\hline Melastomataceae & Miconia cubatanensis Hoehne & 1 & 0,10 & 0,27 & 0,0025 & 0,02 & 0,13 \\
\hline Myrtaceae & Myrcia aethusa (O.Berg) N.Silveira & 1 & 0,10 & 0,27 & 0,0024 & 0,02 & 0,13 \\
\hline Humiriaceae & Sacoglottis mattogrossensis Malme & 1 & 0,10 & 0,27 & 0,0024 & 0,02 & 0,13 \\
\hline Lauraceae & Ocotea lancifolia (Schott) Mez & 1 & 0,10 & 0,27 & 0,0024 & 0,02 & 0,13 \\
\hline Fabaceae & Acosmium lentiscifolium Schott. & 1 & 0,10 & 0,27 & 0,0023 & 0,02 & 0,13 \\
\hline Sapotaceae & Pouteria torta (Mart.) Radlk. & 1 & 0,10 & 0,27 & 0,0023 & 0,02 & 0,13 \\
\hline \multirow[t]{2}{*}{ Ochnaceae } & Ouratea salicifolia (A.St.-Hil. \& Tul.) Engl. & 1 & 0,10 & 0,27 & 0,0022 & 0,02 & 0,13 \\
\hline & Total & 017 & 100 & 100 & 14,20 & 100 & 100 \\
\hline \multicolumn{8}{|l|}{ Grupo II } \\
\hline Euphorbiaceae & Iabea piriri Aubl. & 97 & 12,22 & 2,76 & 0,4070 & 2,84 & 5,94 \\
\hline Chrysobalanaceae & Licania hoehnei Pilg. & 34 & 4,28 & 2,45 & 1,2958 & 9,05 & 5,26 \\
\hline Arecaceae & Attalea oleifera Barb.Rodr. & 14 & 1,76 & 2,76 & 1,4758 & 10,30 & 4,94 \\
\hline Lauraceae & Ocotea corymbosa (Meisn.) & 33 & 4,16 & 2,45 & 0,4854 & 3,39 & 3,33 \\
\hline Rubiaceae & Amaioua guianensis Aubl. & 42 & 5,29 & 2,76 & 0,2768 & 1,93 & 3,33 \\
\hline \multirow[t]{2}{*}{ Sapotaceae } & Chrysophyllum gonocarpum (Mart. \& E & & & & & & \\
\hline & Engl. & 21 & 2,64 & 2,45 & 0,6612 & 4,62 & 3,24 \\
\hline Sapotaceae & Chrysophyllum ma & 21 & 2,64 & 3,07 & 0,4811 & 3,36 & 3,02 \\
\hline Lauraceae & Ocotea aciphylla (Nees \& Mart.) Mez & 19 & 2,39 & 2,15 & 0,5338 & 3,73 & 2,76 \\
\hline \multirow[t]{2}{*}{ Chrysobalanaceae } & Hirtella floribunda Cham. \& Schltdl. & 29 & 3,65 & 2,45 & 0,2717 & 1,90 & 2,67 \\
\hline & Morf & 15 & 1,89 & 1,53 & 0,5114 & 3,57 & 2,33 \\
\hline Fabaceae & Copaifera langsdorffii Desf. & 12 & 1,51 & 2,15 & 0,4219 & 2,95 & 2,20 \\
\hline Arecaceae & Euterpe edulis Mart. & 35 & 4,41 & 0,31 & 0,2666 & 1,86 & 2,19 \\
\hline Myrtaceae & ultiflora (Lam.) DC. & 18 & 2,27 & 1,53 & 0,3120 & 2,18 & 1,99 \\
\hline Myrtaceae & razonica DC. & 20 & 2,52 & 2,45 & 0,1294 & 0,90 & 1,96 \\
\hline Anacardiaceae & Thyrsodium spruceanum Benth. & 17 & 2,14 & 1,53 & 0,2893 & 2,02 & 1,90 \\
\hline Myrtaceae & Eugenia brevistyla D.Legrand & 15 & 1,89 & 2,15 & 0,2001 & 1,40 & 1,81 \\
\hline Lauraceae & Cryptocarya aschersoniana $\mathrm{Mez}$ & 13 & 1,64 & 1,84 & 0,1451 & 1,01 & 1,50 \\
\hline Combretaceae & Buchenavia tetraphylla (Aubl.) R.A.Howard & 4 & 0,50 & 1,23 & 0,3903 & 2,73 & 1,49 \\
\hline Moraceae & Sorocea bonplandii (Baill.) W.C.Burger & 19 & 2,39 & 1,53 & 0,0661 & 0,46 & 1,46 \\
\hline Myrtaceae & Calyptranthes pulchella DC. & 12 & 1,51 & 1,23 & 0,2057 & 1,44 & 1,39 \\
\hline Myrtaceae & lendens (Sw.) DC. & 11 & 1,39 & 2,15 & 0,0886 & 0,62 & 1,38 \\
\hline Combretaceae & Buchenavia tomentosa Eichler & 5 & 0,63 & 1,23 & 0,2924 & 2,04 & 1,30 \\
\hline Fabaceae & Diplotropis ferruginea Benth. & 8 & 1,01 & 1,53 & 0,1875 & 1,31 & 1,28 \\
\hline Rutaceae & Esenbeckia febrifuga (A.St.-Hil.) A & 21 & 2,64 & 0,31 & 0,1261 & 0,88 & 1,28 \\
\hline Nyctaginaceae & Guapira obtusata (Jacq.) Little & 7 & 0,88 & 0,92 & 0,2067 & 1,44 & 1,08 \\
\hline Fabaceae & Inga schinifolia Benth. & 7 & 0,88 & 1,23 & 0,1305 & 0,91 & 1,01 \\
\hline Annonaceae & Duguetia lanceolata A.St.-H & 6 & 0,76 & 0,92 & 0,1843 & 1,29 & 0,99 \\
\hline Myrtaceae & Eugenia capparidifolia DC. & 3 & 0,38 & 0,61 & 0,2787 & 1,95 & 0,98 \\
\hline Elaeocarpaceae & Sloanea monosperma Vell. & 7 & 0,88 & 1,53 & 0,0591 & 0,41 & 0,94 \\
\hline Euphorbiaceae & Alchornea triplinervia (Spreng.) Müll.Arg. & 4 & 0,50 & 0,92 & 0,1908 & 1,33 & 0,92 \\
\hline Annonaceae & Xylopia brasiliensis Spreng. & 7 & 0,88 & 1,53 & 0,0343 & 0,24 & 0,88 \\
\hline Vochysiaceae & Callisthene major Mart. & 11 & 1,39 & 0,31 & 0,1353 & 0,94 & 0,88 \\
\hline Fabaceae & Machaerium nictitans (Vell.) Benth. & 5 & 0,63 & 1,23 & 0,1100 & 0,77 & 0,88 \\
\hline Lauraceae & Ocotea spixiana $(\mathrm{Nees}) \mathrm{Mez}$ & 5 & 0,63 & 1,53 & 0,0531 & 0,37 & 0,84 \\
\hline Calophyllaceae & Kielmeyera sp. & 6 & 0,76 & 0,61 & 0,1461 & 1,02 & 0,80 \\
\hline
\end{tabular}




\begin{tabular}{|c|c|c|c|c|c|c|c|}
\hline Famílias & Espécies & $\mathbf{N i}$ & DR & FR & $\mathbf{A B}$ & DoR & VI\% \\
\hline Sapindaceae & Allophylus edulis (A.St.-Hil. et al.) Hieron. ex Niederl. & 8 & 1,01 & 0,92 & 0,0604 & 0,42 & 0,78 \\
\hline Asteraceae & Piptocarpha macropoda (DC.) Baker & 5 & 0,63 & 1,23 & 0,0661 & 0,46 & 0,77 \\
\hline Myrtaceae & Eugenia florida DC. & 5 & 0,63 & 1,53 & 0,0196 & 0,14 & 0,77 \\
\hline Nyctaginaceae & Guapira opposita (Vell.) Reitz & 5 & 0,63 & 1,23 & 0,0360 & 0,25 & 0,70 \\
\hline Apocynaceae & Aspidosperma cylindrocarpon Müll.Arg. & 5 & 0,63 & 1,23 & 0,0274 & 0,19 & 0,68 \\
\hline Rosaceae & Prunus myrtifolia (L.) Urb. & 6 & 0,76 & 0,92 & 0,0529 & 0,37 & 0,68 \\
\hline Fabaceae & Tachigali friburgensis (Harms) L.G.Silva \& H.C.Lima & 4 & 0,50 & 0,92 & 0,0807 & 0,56 & 0,66 \\
\hline Lecythidaceae & Cariniana estrellensis (Raddi) Kuntze & 1 & 0,13 & 0,31 & 0,2198 & 1,53 & 0,66 \\
\hline Magnoliaceae & Magnolia ovata (A.St.-Hil.) Spreng. & 1 & 0,13 & 0,31 & 0,2193 & 1,53 & 0,65 \\
\hline Meliaceae & Cabralea canjerana (Vell.) Mart. & 3 & 0,38 & 0,61 & 0,1353 & 0,94 & 0,65 \\
\hline Sapotaceae & Pouteria venosa (Mart.) Baehni & 3 & 0,38 & 0,61 & 0,1098 & 0,77 & 0,59 \\
\hline Lauraceae & Persea rufotomentosa Nees \& Mart. & 2 & 0,25 & 0,61 & 0,1243 & 0,87 & 0,58 \\
\hline Annonaceae & Xylopia frutescens Aubl. & 4 & 0,50 & 0,92 & 0,0170 & 0,12 & 0,51 \\
\hline Malvaceae & Luehea divaricata Mart. \& Zucc. & 2 & 0,25 & 0,61 & 0,0941 & 0,66 & 0,51 \\
\hline Myrtaceae & Myrciaria pilosa Sobral \& Couto & 7 & 0,88 & 0,31 & 0,0461 & 0,32 & 0,50 \\
\hline Lauraceae & Nectandra grandiflora Nees & 5 & 0,63 & 0,61 & 0,0300 & 0,21 & 0,48 \\
\hline Boraginaceae & Cordia sellowiana Cham. & 4 & 0,50 & 0,92 & 0,0039 & 0,03 & 0,48 \\
\hline Apocynaceae & Aspidosperma polyneuron Müll.Arg. & 2 & 0,25 & 0,61 & 0,0751 & 0,52 & 0,46 \\
\hline Clusiaceae & Clusia arrudea Planch. \& Triana ex Engl. & 2 & 0,25 & 0,31 & 0,1137 & 0,79 & 0,45 \\
\hline Fabaceae & Plathymenia sp. & 1 & 0,13 & 0,31 & 0,1298 & 0,91 & 0,45 \\
\hline Bignoniaceae & Handroanthus ochraceus (Cham.) Mattos & 2 & 0,25 & 0,61 & 0,0676 & 0,47 & 0,45 \\
\hline Fabaceae & Bauhinia rufa (Bong.) Steud. & 2 & 0,25 & 0,61 & 0,0619 & 0,43 & 0,43 \\
\hline Myrtaceae & Myrcia sp. & 2 & 0,25 & 0,61 & 0,0512 & 0,36 & 0,41 \\
\hline Myrtaceae & Myrcia mischophylla Kiaersk. & 3 & 0,38 & 0,61 & 0,0320 & 0,22 & 0,40 \\
\hline Fabaceae & Melanoxylon brauna Schott & 3 & 0,38 & 0,31 & 0,0758 & 0,53 & 0,40 \\
\hline Sabiaceae & Meliosma sellowii Urb. & 3 & 0,38 & 0,61 & 0,0242 & 0,17 & 0,39 \\
\hline Lauraceae & Ocotea sp. & 2 & 0,25 & 0,61 & 0,0336 & 0,23 & 0,37 \\
\hline Polygonaceae & Coccoloba arborescens (Vell.) R.A.Howard. & 2 & 0,25 & 0,61 & 0,0311 & 0,22 & 0,36 \\
\hline Sapotaceae & Pouteria ramiflora (Mart.) Radlk. & 5 & 0,63 & 0,31 & 0,0190 & 0,13 & 0,36 \\
\hline Calophyllaceae & Calophyllum brasiliense Cambess. & 1 & 0,13 & 0,31 & 0,0892 & 0,62 & 0,35 \\
\hline Celastraceae & Maytenus boaria Molina & 2 & 0,25 & 0,61 & 0,0249 & 0,17 & 0,35 \\
\hline Apocynaceae & Aspidosperma olivaceum Müll.Arg. & 2 & 0,25 & 0,61 & 0,0235 & 0,16 & 0,34 \\
\hline Myrtaceae & Myrcia guianensis (Aubl.) DC. & 4 & 0,50 & 0,31 & 0,0297 & 0,21 & 0,34 \\
\hline Myrtaceae & Eugenia brasiliensis Lam. & 4 & 0,50 & 0,31 & 0,0275 & 0,19 & 0,33 \\
\hline Sapindaceae & Simarouba amara Aubl. & 2 & 0,25 & 0,61 & 0,0191 & 0,13 & 0,33 \\
\hline Lamiaceae & Vitex rufescens A.Juss. & 2 & 0,25 & 0,61 & 0,0191 & 0,13 & 0,33 \\
\hline Euphorbiaceae & Croton rottlerifolius Baill. & 1 & 0,13 & 0,31 & 0,0785 & 0,55 & 0,33 \\
\hline Fabaceae & Holocalyx balansae Micheli & 1 & 0,13 & 0,31 & 0,0741 & 0,52 & 0,32 \\
\hline Bignoniaceae & Handroanthus sp. & 1 & 0,13 & 0,31 & 0,0688 & 0,48 & 0,30 \\
\hline Ochnaceae & Ouratea salicifolia (A.St.-Hil. \& Tul.) Engl. & 2 & 0,25 & 0,61 & 0,0056 & 0,04 & 0,30 \\
\hline Rutaceae & Dictyoloma vandellianum A.Juss. & 2 & 0,25 & 0,61 & 0,0052 & 0,04 & 0,30 \\
\hline Anacardiaceae & Tapirira obtusa (Benth.) J.D.Mitch. & 3 & 0,38 & 0,31 & 0,0286 & 0,20 & 0,29 \\
\hline Apocynaceae & Aspidosperma tomentosum Mart. \& Zucc. & 1 & 0,13 & 0,31 & 0,0584 & 0,41 & 0,28 \\
\hline Fabaceae & Machaerium brasiliense Vogel & 2 & 0,25 & 0,31 & 0,0376 & 0,26 & 0,27 \\
\hline Myrtaceae & Eugenia sp2. & 1 & 0,13 & 0,31 & 0,0539 & 0,38 & 0,27 \\
\hline Polygonaceae & Coccoloba warmingii Meisn. & 2 & 0,25 & 0,31 & 0,0343 & 0,24 & 0,27 \\
\hline Primulaceae & Myrsine coriacea (Sw.) R.Br. ex Roem. \& Schult. & 2 & 0,25 & 0,31 & 0,0288 & 0,20 & 0,25 \\
\hline Lauraceae & Aniba firmula (Nees \& Mart.) Mez & 1 & 0,13 & 0,31 & 0,0411 & 0,29 & 0,24 \\
\hline Fabaceae & Hymenolobium janeirense Kuhlm. & 1 & 0,13 & 0,31 & 0,0401 & 0,28 & 0,24 \\
\hline Connaraceae & Connarus detersus Planch. & 2 & 0,25 & 0,31 & 0,0211 & 0,15 & 0,24 \\
\hline Primulaceae & Cybianthus sp. & 2 & 0,25 & 0,31 & 0,0148 & 0,10 & 0,22 \\
\hline Myrtaceae & Pimenta pseudocaryophyllus (Gomes) Landrum & 2 & 0,25 & 0,31 & 0,0143 & 0,10 & 0,22 \\
\hline Myrtaceae & Psidium sp1. & 1 & 0,13 & 0,31 & 0,0319 & 0,22 & 0,22 \\
\hline Myrtaceae & Calyptranthes clusiifolia O.Berg & 2 & 0,25 & 0,31 & 0,0083 & 0,06 & 0,21 \\
\hline Sapindaceae & Cupania rubiginosa (Poir.) Radlk. & 2 & 0,25 & 0,31 & 0,0059 & 0,04 & 0,20 \\
\hline Rubiaceae & Psychotria vellosiana Benth. & 2 & 0,25 & 0,31 & 0,0047 & 0,03 & 0,20 \\
\hline Myrtaceae & Eugenia sp. & 2 & 0,25 & 0,31 & 0,0040 & 0,03 & 0,20 \\
\hline Lauraceae & Nectandra sp. & 1 & 0,13 & 0,31 & 0,0214 & 0,15 & 0,19 \\
\hline Myrtaceae & Myrcia sp2. & 1 & 0,13 & 0,31 & 0,0209 & 0,15 & 0,19 \\
\hline Salicaceae & Casearia cubensis Urb & 1 & 0,13 & 0,31 & 0,0205 & 0,14 & 0,19 \\
\hline Fabaceae & Copaifera oblongifolia Mart. ex Hayne & 1 & 0,13 & 0,31 & 0,0198 & 0,14 & 0,19 \\
\hline
\end{tabular}




\begin{tabular}{|c|c|c|c|c|c|c|c|}
\hline Famílias & Espécies & $\mathbf{N i}$ & DR & FR & $\mathbf{A B}$ & DoR & VI\% \\
\hline Malphiguiaceae & Byrsonima chrysophylla Kunth & 1 & 0,13 & 0,31 & 0,0194 & 0,14 & 0,19 \\
\hline Lamiaceae & Vitex polygama Cham. & 1 & 0,13 & 0,31 & 0,0178 & 0,12 & 0,19 \\
\hline Vochysiaceae & Qualea multiflora Mart. & 1 & 0,13 & 0,31 & 0,0161 & 0,11 & 0,18 \\
\hline Myrtaceae & Myrcia mutabilis (O.Berg) N.Silveira & 1 & 0,13 & 0,31 & 0,0126 & 0,09 & 0,17 \\
\hline Myrtaceae & Psidium rufum Mart. ex DC. & 1 & 0,13 & 0,31 & 0,0117 & 0,08 & 0,17 \\
\hline Moraceae & Brosimum glaziovii Taub. & 1 & 0,13 & 0,31 & 0,0107 & 0,07 & 0,17 \\
\hline Euphorbiaceae & Alchornea glandulosa Poepp. \& Endl. & 1 & 0,13 & 0,31 & 0,0105 & 0,07 & 0,17 \\
\hline Aquifoliaceae & Ilex brevicuspis Reissek & 1 & 0,13 & 0,31 & 0,0103 & 0,07 & 0,17 \\
\hline Fabaceae & Tachigali paratyensis (Vell.) H.C.Lima & 1 & 0,13 & 0,31 & 0,0087 & 0,06 & 0,16 \\
\hline Myrtaceae & Myrcia coelosepala Kiaersk & 1 & 0,13 & 0,31 & 0,0080 & 0,06 & 0,16 \\
\hline Theaceae & Laplacea fruticosa (Schrad.) Kobuski & 1 & 0,13 & 0,31 & 0,0076 & 0,05 & 0,16 \\
\hline Fabaceae & Swartzia apetala Raddi & 1 & 0,13 & 0,31 & 0,0076 & 0,05 & 0,16 \\
\hline Myrtaceae & Eugenia cerasiflora Miq. & 1 & 0,13 & 0,31 & 0,0075 & 0,05 & 0,16 \\
\hline Meliaceae & Cedrela odorata L. & 1 & 0,13 & 0,31 & 0,0074 & 0,05 & 0,16 \\
\hline Melastomataceae & Miconia sp. & 1 & 0,13 & 0,31 & 0,0067 & 0,05 & 0,16 \\
\hline Elaeocarpaceae & Sloanea guianensis (Aubl.) Benth. & 1 & 0,13 & 0,31 & 0,0067 & 0,05 & 0,16 \\
\hline Sapotaceae & Chrysophyllum inornatum Mart. & 1 & 0,13 & 0,31 & 0,0060 & 0,04 & 0,16 \\
\hline Celastraceae & Monteverdia floribunda (Reissek) Biral & 1 & 0,13 & 0,31 & 0,0055 & 0,04 & 0,16 \\
\hline Ochnaceae & Ouratea castaneifolia (DC.) Engl. & 1 & 0,13 & 0,31 & 0,0050 & 0,04 & 0,16 \\
\hline Proteaceae & Roupala montana Aubl. & 1 & 0,13 & 0,31 & 0,0047 & 0,03 & 0,16 \\
\hline Rubiaceae & Faramea sp. & 1 & 0,13 & 0,31 & 0,0044 & 0,03 & 0,15 \\
\hline Lauraceae & Nectandra lanceolata Nees & 1 & 0,13 & 0,31 & 0,0042 & 0,03 & 0,15 \\
\hline Myrtaceae & Myrcia florida Lem. & 1 & 0,13 & 0,31 & 0,0040 & 0,03 & 0,15 \\
\hline Sapotaceae & Pouteria torta (Mart.) Radlk. & 1 & 0,13 & 0,31 & 0,0040 & 0,03 & 0,15 \\
\hline Salicaceae & Casearia decandra Jacq. & 1 & 0,13 & 0,31 & 0,0033 & 0,02 & 0,15 \\
\hline Lauraceae & Ocotea silvestris Vattimo-Gil & 1 & 0,13 & 0,31 & 0,0029 & 0,02 & 0,15 \\
\hline Melastomataceae & Miconia francavillana Cogn. & 1 & 0,13 & 0,31 & 0,0028 & 0,02 & 0,15 \\
\hline Myrtaceae & Myrcia tomentosa (Aubl.) DC. & 1 & 0,13 & 0,31 & 0,0028 & 0,02 & 0,15 \\
\hline Salicaceae & Casearia cf. decandra Jacq. & 1 & 0,13 & 0,31 & 0,0027 & 0,02 & 0,15 \\
\hline Lauraceae & Nectandra membranacea (Sw.) Griseb. & 1 & 0,13 & 0,31 & 0,0027 & 0,02 & 0,15 \\
\hline Apocynaceae & Aspidosperma ramiflorum Müll.Arg. & 1 & 0,13 & 0,31 & 0,0026 & 0,02 & 0,15 \\
\hline Myrtaceae & Eugenia sonderiana O.Berg & 1 & 0,13 & 0,31 & 0,0025 & 0,02 & 0,15 \\
\hline Annonaceae & Guatteria australis A.St.-Hil. & 1 & 0,13 & 0,31 & 0,0023 & 0,02 & 0,15 \\
\hline Melastomataceae & Miconia cubatanensis Hoehne & 1 & 0,13 & 0,31 & 0,0023 & 0,02 & 0,15 \\
\hline Symplocaceae & Symplocos platyphylla (Pohl) Benth. & 1 & 0,13 & 0,31 & 0,0023 & 0,02 & 0,15 \\
\hline \multirow[t]{2}{*}{ Melastomataceae } & Tibouchina cerastifolia Cogn. & 1 & 0,13 & 0,31 & 0,0021 & 0,01 & 0,15 \\
\hline & Total Geral & 794 & 100 & 100 & 14,32 & 100 & 100 \\
\hline \multicolumn{8}{|l|}{ Grupo III } \\
\hline Sapindaceae & Allophylus edulis (A.St.-Hil. et al.) Hieron. ex Niederl. & 36 & 9,57 & 2,38 & 0,5223 & 7,49 & 6,48 \\
\hline Anacardiaceae & Thyrsodium spruceanum Benth. & 21 & 5,59 & 2,98 & 0,3054 & 4,38 & 4,31 \\
\hline Myrtaceae & Myrcia multiflora (Lam.) DC. & 11 & 2,93 & 2,38 & 0,5193 & 7,45 & 4,25 \\
\hline Chrysobalanaceae & Licania hoehnei Pilg. & 10 & 2,66 & 2,38 & 0,4149 & 5,95 & 3,66 \\
\hline Arecaceae & Attalea oleifera Barb.Rodr. & 4 & 1,06 & 1,19 & 0,5796 & 8,32 & 3,52 \\
\hline Myrtaceae & Myrcia amazonica DC. & 21 & 5,59 & 2,38 & 0,0966 & 1,39 & 3,12 \\
\hline Calophyllaceae & Kielmeyera sp. & 11 & 2,93 & 2,38 & 0,2723 & 3,91 & 3,07 \\
\hline Lauraceae & Ocotea corymbosa (Meisn.) Mez & 10 & 2,66 & 1,19 & 0,2534 & 3,64 & 2,50 \\
\hline Myrtaceae & Myrcia splendens (Sw.) DC. & 12 & 3,19 & 2,38 & 0,0710 & 1,02 & 2,20 \\
\hline \multirow[t]{2}{*}{ Sapotaceae } & Chrysophyllum marginatum (Hook. \& Arn.) Radlk. & 7 & 1,86 & 1,79 & 0,1852 & 2,66 & 2,10 \\
\hline & Morfotipo & 9 & 2,39 & 1,79 & 0,1387 & 1,99 & 2,06 \\
\hline Chrysobalanaceae & Hirtella floribunda Cham. \& Schltdl. & 8 & 2,13 & 1,79 & 0,1452 & 2,08 & 2,00 \\
\hline Salicaceae & Casearia decandra Jacq. & 7 & 1,86 & 2,38 & 0,1170 & 1,68 & 1,97 \\
\hline Lauraceae & Ocotea spixiana (Nees) Mez & 7 & 1,86 & 2,38 & 0,0966 & 1,39 & 1,88 \\
\hline Myrtaceae & Myrcia mischophylla Kiaersk. & 6 & 1,60 & 1,79 & 0,1232 & 1,77 & 1,72 \\
\hline Fabaceae & Copaifera langsdorffii Desf. & 4 & 1,06 & 1,79 & 0,1419 & 2,04 & 1,63 \\
\hline Rubiaceae & Amaioua guianensis Aubl. & 7 & 1,86 & 1,79 & 0,0671 & 0,96 & 1,54 \\
\hline Meliaceae & Cabralea canjerana (Vell.) Mart. & 4 & 1,06 & 1,19 & 0,1584 & 2,27 & 1,51 \\
\hline Euphorbiaceae & Mabea piriri Aubl. & 10 & 2,66 & 1,19 & 0,0465 & 0,67 & 1,51 \\
\hline Lauraceae & Ocotea aciphylla (Nees \& Mart.) Mez & 5 & 1,33 & 1,19 & 0,1253 & 1,80 & 1,44 \\
\hline Fabaceae & Plathymenia sp. & 3 & 0,80 & 1,19 & 0,1556 & 2,23 & 1,41 \\
\hline Fabaceae & Diplotropis ferruginea Benth. & 8 & 2,13 & 1,19 & 0,0619 & 0,89 & 1,40 \\
\hline
\end{tabular}




\begin{tabular}{|c|c|c|c|c|c|c|c|}
\hline Famílias & Espécies & $\mathbf{N i}$ & DR & FR & $\mathbf{A B}$ & DoR & VI\% \\
\hline Lauraceae & Ocotea $\mathrm{sp}$. & 5 & 1,33 & 1,79 & 0,0715 & 1,03 & 1,38 \\
\hline Simaroubaceae & Simarouba amara Aubl. & 5 & 1,33 & 1,79 & 0,0680 & 0,98 & 1,36 \\
\hline Elaeocarpaceae & Sloanea monosperma Vell. & 6 & 1,60 & 1,79 & 0,0442 & 0,63 & 1,34 \\
\hline Fabaceae & Machaerium nictitans (Vell.) Benth. & 5 & 1,33 & 1,19 & 0,0894 & 1,28 & 1,27 \\
\hline Apocynaceae & Aspidosperma tomentosum Mart. \& Zucc. & 1 & 0,27 & 0,60 & 0,1718 & 2,46 & 1,11 \\
\hline Combretaceae & Buchenavia tomentosa Eichler & 3 & 0,80 & 1,19 & 0,0852 & 1,22 & 1,07 \\
\hline Apocynaceae & Aspidosperma illustre (Vell.) Kuhlm. \& Pirajá & 3 & 0,80 & 0,60 & 0,1218 & 1,75 & 1,05 \\
\hline Myrtaceae & Calyptranthes pulchella DC. & 2 & 0,53 & 1,19 & 0,0983 & 1,41 & 1,04 \\
\hline Bignoniaceae & Handroanthus ochraceus (Cham.) Mattos & 3 & 0,80 & 0,60 & 0,1053 & 1,51 & 0,97 \\
\hline Myrtaceae & Myrcia guianensis (Aubl.) DC. & 3 & 0,80 & 1,79 & 0,0212 & 0,30 & 0,96 \\
\hline Lauraceae & Aniba firmula (Nees \& Mart.) Mez & 1 & 0,27 & 0,60 & 0,1376 & 1,97 & 0,95 \\
\hline Malvaceae & Luehea divaricata Mart. \& Zucc. & 3 & 0,80 & 0,60 & 0,0878 & 1,26 & 0,88 \\
\hline Lauraceae & Ocotea glaziovii $\mathrm{Mez}$ & 3 & 0,80 & 1,19 & 0,0401 & 0,58 & 0,85 \\
\hline Meliaceae & Trichilia pallens C.DC. & 5 & 1,33 & 0,60 & 0,0436 & 0,63 & 0,85 \\
\hline Lauraceae & Nectandra grandiflora Nees & 4 & 1,06 & 1,19 & 0,0186 & 0,27 & 0,84 \\
\hline Salicaceae & Casearia cubensis Urb & 2 & 0,53 & 1,19 & 0,0511 & 0,73 & 0,82 \\
\hline Moraceae & Sorocea bonplandii (Baill.) W.C.Burger & 4 & 1,06 & 1,19 & 0,0140 & 0,20 & 0,82 \\
\hline Fabaceae & Andira anthelmia (Vell.) Benth. & 4 & 1,06 & 1,19 & 0,0121 & 0,17 & 0,81 \\
\hline Lecythidaceae & Cariniana estrellensis (Raddi) Kuntze & 1 & 0,27 & 0,60 & 0,1043 & 1,50 & 0,79 \\
\hline Fabaceae & Dalbergia foliolosa Benth. & 2 & 0,53 & 1,19 & 0,0392 & 0,56 & 0,76 \\
\hline Fabaceae & Pterogyne nitens Tul. & 4 & 1,06 & 0,60 & 0,0409 & 0,59 & 0,75 \\
\hline Celastraceae & Maytenus robusta Reissek & 3 & 0,80 & 1,19 & 0,0142 & 0,20 & 0,73 \\
\hline Fabaceae & Inga marginata Willd. & 2 & 0,53 & 0,60 & 0,0726 & 1,04 & 0,72 \\
\hline Myrtaceae & Calyptranthes clusiifolia O.Berg & 2 & 0,53 & 1,19 & 0,0238 & 0,34 & 0,69 \\
\hline Melastomataceae & Mouriri glazioviana Cogn. & 4 & 1,06 & 0,60 & 0,0267 & 0,38 & 0,68 \\
\hline Boraginaceae & Cordia ecalyculata Vell. & 2 & 0,53 & 0,60 & 0,0620 & 0,89 & 0,67 \\
\hline Myrtaceae & Myrcia mutabilis (O.Berg) N.Silveira & 2 & 0,53 & 1,19 & 0,0174 & 0,25 & 0,66 \\
\hline Lauraceae & Cryptocarya aschersoniana $\mathrm{Mez}$ & 2 & 0,53 & 1,19 & 0,0117 & 0,17 & 0,63 \\
\hline Lamiaceae & Vitex polygama Cham. & 2 & 0,53 & 1,19 & 0,0099 & 0,14 & 0,62 \\
\hline Primulaceae & Cybianthus sp. & 3 & 0,80 & 0,60 & 0,0314 & 0,45 & 0,61 \\
\hline Sapindaceae & Cupania vernalis Cambess. & 2 & 0,53 & 1,19 & 0,0056 & 0,08 & 0,60 \\
\hline Myrtaceae & Psidium rufum Mart. ex DC. & 2 & 0,53 & 0,60 & 0,0427 & 0,61 & 0,58 \\
\hline Combretaceae & Buchenavia tetraphylla (Aubl.) R.A.Howard & 1 & 0,27 & 0,60 & 0,0579 & 0,83 & 0,56 \\
\hline Lauraceae & Nectandra membranacea (Sw.) Griseb. & 3 & 0,80 & 0,60 & 0,0185 & 0,27 & 0,55 \\
\hline Proteaceae & Roupala montana Aubl. & 3 & 0,80 & 0,60 & 0,0095 & 0,14 & 0,51 \\
\hline Sapotaceae & Pouteria venosa (Mart.) Baehni & 2 & 0,53 & 0,60 & 0,0246 & 0,35 & 0,49 \\
\hline Fabaceae & Dalbergia brasiliensis Vogel & 2 & 0,53 & 0,60 & 0,0243 & 0,35 & 0,49 \\
\hline Fabaceae & Melanoxylon brauna Schott & 1 & 0,27 & 0,60 & 0,0416 & 0,60 & 0,49 \\
\hline Perceae & Pera glabrata (Schott) Baill. & 2 & 0,53 & 0,60 & 0,0230 & 0,33 & 0,49 \\
\hline \multirow[t]{2}{*}{ Fabaceae } & Senna macranthera (DC. ex Collad.) H.S.Irwin \& & & & & & & \\
\hline & Barneby & 2 & 0,53 & 0,60 & 0,0177 & 0,25 & 0,46 \\
\hline Myrtaceae & Myrcia sp. & 2 & 0,53 & 0,60 & 0,0167 & 0,24 & 0,46 \\
\hline Myrtaceae & Eugenia prasina O.Berg & 1 & 0,27 & 0,60 & 0,0333 & 0,48 & 0,45 \\
\hline Symplocaceae & Symplocos platyphylla (Pohl) Benth. & 2 & 0,53 & 0,60 & 0,0143 & 0,20 & 0,44 \\
\hline Fabaceae & Swartzia apetala Raddi & 2 & 0,53 & 0,60 & 0,0109 & 0,16 & 0,43 \\
\hline Anacardiaceae & Tapirira obtusa (Benth.) J.D.Mitch. & 2 & 0,53 & 0,60 & 0,0105 & 0,15 & 0,43 \\
\hline Annonaceae & Xylopia frutescens Aubl. & 2 & 0,53 & 0,60 & 0,0102 & 0,15 & 0,42 \\
\hline Fabaceae & Holocalyx balansae Micheli & 2 & 0,53 & 0,60 & 0,0098 & 0,14 & 0,42 \\
\hline Sapotaceae & Pouteria torta (Mart.) Radlk. & 1 & 0,27 & 0,60 & 0,0269 & 0,39 & 0,42 \\
\hline Annonaceae & Duguetia chrysocarpa Maas & 2 & 0,53 & 0,60 & 0,0071 & 0,10 & 0,41 \\
\hline Nyctaginaceae & Guapira opposita (Vell.) Reitz & 2 & 0,53 & 0,60 & 0,0069 & 0,10 & 0,41 \\
\hline Lauraceae & Cinnamomum triplinerve (Ruiz \& Pav.) Kosterm. & 1 & 0,27 & 0,60 & 0,0232 & 0,33 & 0,40 \\
\hline Moraceae & Ficus mexiae Standl. & 1 & 0,27 & 0,60 & 0,0215 & 0,31 & 0,39 \\
\hline Theaceae & Laplacea Kunth & 1 & 0,27 & 0,60 & 0,0210 & 0,30 & 0,39 \\
\hline Annonaceae & Guatteria australis A.St.-Hil. & 1 & 0,27 & 0,60 & 0,0184 & 0,26 & 0,38 \\
\hline Myrtaceae & Myrcia coelosepala Kiaersk & 1 & 0,27 & 0,60 & 0,0165 & 0,24 & 0,37 \\
\hline Myrtaceae & Eugenia capparidifolia DC. & 1 & 0,27 & 0,60 & 0,0142 & 0,20 & 0,35 \\
\hline Meliaceae & Guarea macrophylla Vahl & 1 & 0,27 & 0,60 & 0,0130 & 0,19 & 0,35 \\
\hline Lauraceae & Ocotea silvestris Vattimo-Gil & 1 & 0,27 & 0,60 & 0,0121 & 0,17 & 0,34 \\
\hline Theaceae & Laplacea fruticosa (Schrad.) Kobuski & 1 & 0,27 & 0,60 & 0,0104 & 0,15 & 0,34 \\
\hline Myrtaceae & Myrcia pubipetala Miq. & 1 & 0,27 & 0,60 & 0,0089 & 0,13 & 0,33 \\
\hline
\end{tabular}




\begin{tabular}{llrrrrrr}
\hline Famílias & Espécies & Ni & DR & FR & AB & DoR & VI\% \\
\hline Annonaceae & Duguetia lanceolata A.St.-Hil. & 1 & 0,27 & 0,60 & 0,0071 & 0,10 & 0,32 \\
Fabaceae & Machaerium hirtum (Vell.) Stellfeld & 1 & 0,27 & 0,60 & 0,0065 & 0,09 & 0,32 \\
Myrtaceae & Psidium sp. & 1 & 0,27 & 0,60 & 0,0064 & 0,09 & 0,32 \\
Sabiaceae & Meliosma sellowii Urb. & 1 & 0,27 & 0,60 & 0,0057 & 0,08 & 0,31 \\
Euphorbiaceae & Sapium glandulosum (L.) Morong & 1 & 0,27 & 0,60 & 0,0049 & 0,07 & 0,31 \\
Rubiaceae & Cordiera concolor (Cham.) Kuntze & 1 & 0,27 & 0,60 & 0,0045 & 0,06 & 0,31 \\
Polygonaceae & Coccoloba warmingii Meisn. & 1 & 0,27 & 0,60 & 0,0040 & 0,06 & 0,31 \\
Annonaceae & Xylopia brasiliensis Spreng. & 1 & 0,27 & 0,60 & 0,0036 & 0,05 & 0,30 \\
Sapotaceae & Chrysophyllum gonocarpum (Mart. \& Eichler ex Miq.) & & & & & & \\
& Engl. & 1 & 0,27 & 0,60 & 0,0035 & 0,05 & 0,30 \\
Salicaceae & Casearia lasiophylla Eichler & 1 & 0,27 & 0,60 & 0,0032 & 0,05 & 0,30 \\
Ochnaceae & Ouratea castaneifolia (DC.) Engl. & 1 & 0,27 & 0,60 & 0,0031 & 0,04 & 0,30 \\
Fabaceae & Inga schinifolia Benth. & 1 & 0,27 & 0,60 & 0,0030 & 0,04 & 0,30 \\
Sapindaceae & Matayba elaeagnoides Radlk. & 1 & 0,27 & 0,60 & 0,0028 & 0,04 & 0,30 \\
Annonaceae & Xylopia laevigata (Mart.) R.E.Fr. & 1 & 0,27 & 0,60 & 0,0022 & 0,03 & 0,30 \\
Melastomataceae & Tibouchina cerastifolia Cogn. & 1 & 0,27 & 0,60 & 0,0021 & 0,03 & 0,30 \\
Lauraceae & Nectandra oppositifolia Nees & 1 & 0,27 & 0,60 & 0,0020 & 0,03 & 0,30 \\
\hline & Total & $\mathbf{3 7 6}$ & $\mathbf{1 0 0}$ & $\mathbf{1 0 0}$ & $\mathbf{6 , 9 6 8 6}$ & $\mathbf{1 0 0}$ & $\mathbf{1 0 0}$ \\
\hline
\end{tabular}

Em que: $\mathrm{Ni}=$ número de indivíduos, $\mathrm{DR}=$ Densidade Relativa $(\%) ; \mathrm{FR}=$ Frequência Relativa $(\%) ; \mathrm{AB}=$ Área Basal $\left(\mathrm{m}^{2}\right) ;$ DoR $=$ Dominância Relativa $(\%)$; VI = Valor de Importância.

No grupo II as espécies Mabea piriri, Licania hoehnei, Attalea oleifera, Ocotea corymbosa, Amaioua guianensis, Chrysophyllum gonocarpum, Chrysophyllum marginatum, Ocotea aciphylla, Hirtella floribunda e morfotipo contribuíram com $(40,9 \%)$ da densidade total de indivíduos, obtiveram as maiores frequências, área basal, dominância relativa e valor de importância. Os gêneros que apresentaram o maior número de espécies foram Myrcia (11), Eugenia (8) Aspidosperma (5) e Ocotea com (5) espécies.

No grupo III as espécies Allophylus edulis, Thyrsodium spruceanum, Myrcia multiflora, Licania hoehnei, Attalea oleifera, Myrcia amazonica, Kielmeyera sp., Ocotea corymbosa, Myrcia splendens e Chrysophyllum marginatum contribuíram com $38 \%$ da densidade total de indivíduos. Além disso, estas apresentaram maiores frequências, área basal, dominância relativa e valor de importância (Tabela 2). Os gêneros que apresentaram o maior número de espécies foram Myrcia e Ocotea com nove e seis espécies, respectivamente.

As espécies de maior valor de importância encontradas nos grupos I e II foram Licania hoehnei, Ocotea corymbosa, Mabea piriri, Ocotea aciphylla e Amaioua guianensis. As espécies encontradas entre os grupos I e III Licania hoehnei, Ocotea corymbosa e Thyrsodium spruceanum e entre os grupos II e III foram às espécies Licania hoehnei, Attalea oleífera, Ocotea corymbosa e Chrysophyllum marginatum. As espécies Licania hoehnei e Ocotea corymbosa são amplamente distribuídas na comunidade, pois, são encontradas nos três grupos.

As espécies que se destacaram pelo valor de importância (VI) nos três grupos, são diferentes das encontradas em outros estudos na Serra do Espinhaço [2], demonstrando uma rica diversidade florística encontrada nas Florestas Estacionais Semideciduais - FESD. Nos três grupos 5,3\%, $6,5 \%$ e $8,7 \%$ das espécies possuem baixa densidade, pois foram amostrados um único indivíduo cada (Tabela 2). Segundo Caiafa e Martins (2010) [51] são consideradas como espécies raras, pois, apresentaram baixa capacidade de colonização, ou devido competição interespecífica dessas com as espécies já estabelecidas e melhor adaptadas. Foram observados também que algumas espécies se encontram na lista vermelha de espécies ameaçadas de extinção, como: Cariniana estrellensis (em perigo), Melanoxylon brauna, Cedrela odorata e Euterpe edulis (vulnerável) (CNCFLORA), todas com baixa densidade populacional.

A maioria dos indivíduos se encontram na menor classe diamétrica I - 57,5\%, II - 55,6\% e III $-50,5 \%$. Costa et al. (2020) [2] encontraram resultado similar em uma FESD no Parque Estadual do Biribiri, Minas Gerais, destacando acentuado decréscimo na frequência de indivíduos com o aumento das classes. A distribuição diamétrica da comunidade foi representada por um modelo de sucessão do tipo J-invertido (Figura 5), que é típico de florestas naturais com populações autorregenerantes $[33,52,53]$. 


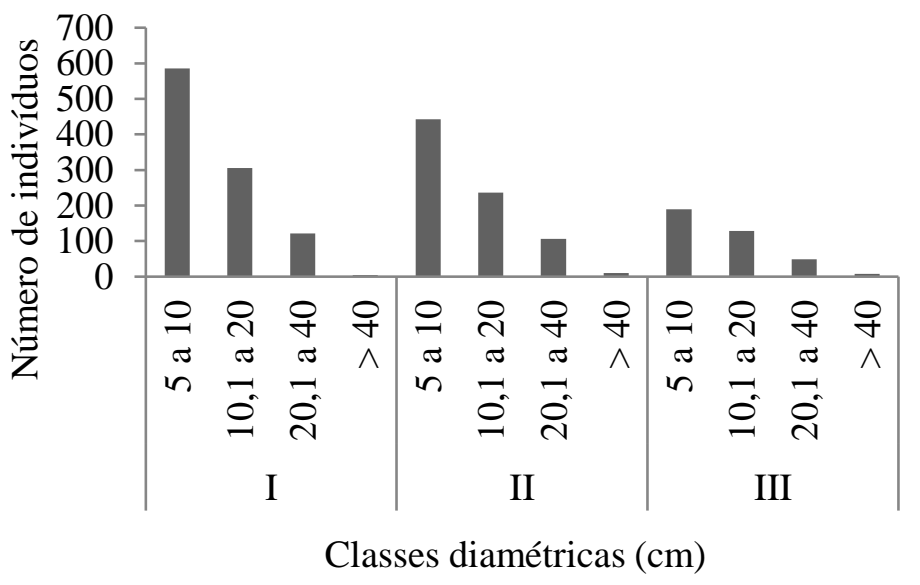

Figura 5. Distribuição diamétrica dos indivíduos na Mata do Gavião, Parque Nacional das SempreVivas, Minas Gerais, grupo I; grupo II e grupo III.

Mesmo com intervalo maior a partir da segunda classe, a maior frequência de indivíduos ocorreu na primeira (Figura 5), indicando que esse fragmento está em fase de construção avançando no processo sucessional $[14,54]$. A distribuição no formato exponencial negativo é comum em florestas heterogêneas, que tendem ao equilíbrio entre mortalidade e recrutamento de indivíduos, com uma renovação natural do estoque de plantas jovens capaz de substituir as adultas. Além disso, muitas espécies não alcançam diâmetros maiores, que é uma característica natural da sua própria fisiologia [46].

\section{CONCLUSÃO}

Os fatores ambientais e espaciais atuam nos padrões de distribuição das espécies no fragmento de Floresta Estacional Semidecidual, localizado no Parque Nacional das Sempre-Vivas, na Reserva da Biosfera da Serra do Espinhaço.

Os resultados mostraram que é possível separar grupos de parcelas em função das variáveis ambientais. $\mathrm{Na}$ estruturação dessa comunidade, parece haver uma interação entre o conjunto das variáveis mensuradas com outras não incluídas na análise, possivelmente devem explicar em maior parte essa relação.

Essa comunidade possui alta diversidade florística e ausência de dominância ecológica, e possui espécies ameaçadas de extinção. A estrutura da comunidade mostra que ela possui indivíduos em todas as classes diamétricas, no entanto foi observado a diminuição do número de indivíduos em função do aumento das classes diamétricas, indica que essa comunidade possui condições para o avanço sucessional.

\section{AGRADECIMENTOS}

Ao Programa de Pós-Graduação em Ciência Florestal da Universidade Federal dos Vales do Jequitinhonha e Mucuri. Aos colaboradores que contribuíram para a realização dessa pesquisa.

\section{REFERÊNCIAS BIBLIOGRÁFICAS}

1. Terra MCNS, Mello JM, Mello CR, Santos RM, Nunes ACR, Raimundo MR. Influência topo-edafoclimática na vegetação de um fragmento de Mata Atlântica na Serra da Mantiqueira, MG. Rev Ambient Água. 2015 Oct/Dec;10(4):1-15. doi: 10.4136/ambi-agua.1705

2. Costa TR, Moura CC, Silva LS, Fonseca DC, Otoni TJO, Machado ELM. Estrutura e relação vegetaçãoambiente de uma Floresta Estacional Semidecidual na Reserva da Biosfera da Serra do Espinhaço. Rev Vozes dos Vales - UFVJM - MG - Brasil. 2020 Out;18(1):1-25. 
3. Maçaneiro JP, Oliveira LZ, Seubert RC, Eisenlohr PV, Schorn LA. More than environmental control at local scales: do spatial processes play an important role in floristic variation in subtropical forests? Acta Bot Brasilica. 2016 Apr-Jun;30(2):183-92. doi: 10.1590/0102-33062015abb0294

4. Sanchez M, Pedroni F, Eisenlohr PV, Oliveira-Filho AT. Changes in tree community composition and structure of Atlantic rain forest on a slope of the Serra do Mar range, Southeastern Brazil, from near sea level to 1000 m of altitude. Rev Flora. 2013 Mar;208(3):184-96. doi: 10.1016/j.flora.2013.03.002

5. Oliveira-Filho AT, Budke JC, Jarenkow JA, Eisenlohr PV, Neves DRM. Delving into the variations in tree species composition and richness across South American subtropical Atlantic and Pampean forests. J Plant Ecol. 2015 Jun;8(3):242-60. doi: 10.1093/jpe/rtt058

6. Begon M, Townsend CR, Harper JL. Ecology: from individuals to ecosystems. Oxford (GB): Blakwell Publishing; 2006.

7. Honrado J, Gonçalves J, Lomba A, Vicente J. Ecologia da paisagem e biodiversidade: da investigação à gestão e à conservação. Rev Online Socied Port Ecol. 2012 Jan;5:36-51.

8. Baldeck CA, Harms KE, Yavitt JB, John R, Turner BL, Valencia R, et al. Soil resources and topography shape local tree community structure in tropical forests. Proc Royal Soc B. 2013 Feb;280(1753):20122532. doi: 10.1098/rspb.2012.2532

9. Hubbell SP. The Unified Neutral Theory of Biodiversity and Biogeography. Princeton (NJ): Princeton University Press; 2001.

10. Magnago LFS, Edwards DP, Edwards FA, Magrach A, Martins SV, Laurance WF. Functional attributes change but functional richness is unchanged after fragmentation of Brazilian Atlantic forests. $\mathrm{J}$ Ecol. 2014 Dec;102:475-85. doi: 10.1111/1365-2745.12206

11. Socolar JB, Gilroy JJ, KuninWE, Edwards DP. How should beta-diversity inform biodiversity conservation? Trends Ecol Evol. 2016 Dec;31:67-80. doi: 10.1016/j.tree.2015.11.005

12. Saporetti Junior AW, Ferreira Júnior WG, Menezes LFT, Martins SV. Estrutura e grupos ecológicos de um trecho de floresta estacional semidecidual montana no município de Dom Silvério, Minas Gerais. Rev Interf. 2016 Dez;12:55-69.

13. Dexter KG, Pennington RT, Oliveira-Filho AT, Bueno ML, Miranda PLS, Neves DM. Inserting Tropical Dry Forests into the discussion on Biome Transitions in the Tropics. Front Ecol. 2018 Jul;6:114. doi: $10.3389 /$ fevo.2018.00104

14. Pereira IM, Gonzaga APD, Machado ELM, Oliveira MLR, Marques IC. Estrutura da vegetação colonizadora em ambiente degradado por extração de cascalho em Diamantina, MG. Pesq Fl Bras. Abr 2015;35(82):77-88. doi: 10.4336/2015.pfb.35.82.769

15. Andrade MA, Martins CS, Domingues SA, organizadores. Reserva da Biosfera da Serra do Espinhaço Primeira Revisão Periódica. 1. ed. Belo Horizonte (MG): MaBUNESCO; 2015.

16. Menezes ES, Mucida DP, França LCJ, Aguilar MVM, Pereira IM. Análise temporal de focos de calor na reserva da biosfera da Serra do Espinhaço. Nativa. 2019 Mai/Jun;7(3):256-61. doi: 10.31413/nativa.v7i3.6877

17. Araújo RW. Geoambientes e relação solo-vegetação no Parque Nacional DAS Sempre-Vivas, Minas Gerais, Brasil [dissertação]. Viçosa (MG): Universidade Federal de Viçosa; 2015. 115 p.

18. Brasil. Ministério da Agricultura Pecuária e Abastecimento. Instituto Nacional de Meteorologia (INMET) [Internet]; sem data de publicação [citado em 08 ago 2020]. Disponível em: http://www.inmet.gov.br

19. Angiosperm Phylogeny Group (APG IV). An update of the Angiosperm Phylogeny Group classification for the orders and families of flowering plants: APG IV. Botanical J Linnean Soc. 2016 May;181(1):1 20. doi: 10.1111/boj.12385

20. Da Silva FC, editor. Manual de análises químicas de solos, plantas e fertilizantes. 2. ed. Brasília (DF): Embrapa Informação Tecnológica; Rio de Janeiro (RJ): Embrapa Solos; 2009.

21. Teixeira PC, Donagemma GK, Fontana A, Teixeira WG, editores. Manual de métodos de análise de solo [Internet]. 3. ed. rev. e ampl. Brasília (DF): Embrapa; 2017 [citado em 08 ago 2020]. Disponível em: https://www.embrapa.br/busca-de-publicacoes/-/publicacao/1085209/manual-de-metodos-deanalise-de-solo

22. Dos Santos HG, Jacomine PKT, Dos Anjos LHC, De Oliveira VA, Lumbreras JF, Coelho MR, et al. Sistema Brasileiro de Classificação de Solos. 5. ed. rev. e ampl. Brasília (DF): Embrapa; 2018.

23. Vieira AD. Diversidade alfa e padrões estruturais de uma Floresta Estacional Semidecidual no Alto Jequitinhonha [dissertação]. Diamantina (MG): Universidade Federal dos Vales do Jequitinhonha e Mucuri; 2015. 63 p.

24. Blanchet FG, Legendre P, Borcard D. Forward selection of explanatory variables. Ecology. 2008 Sep;89(9):2623-32. doi: 10.1890/07-0986.1

25. R Core Team. R: A language and environment for statistical computing. Vienna (AT): R Foundation for Statistical Computing; 2018 [citado em 08 ago 2020]. Disponível em: https://www.r-project.org/ 
26. Oksanen J, Blanchet FG, Friendly M, Kindt R, Legendre P, Mcglinn D, et al. 2017. vegan: Community Ecology Package. R package version 2.4-0; 2020 [acessado em 05 ago 2020]. Disponível em: https://CRAN.R-project.org/package=vegan

27. Dray S, DufourAB. The ade4 package: implementing the duality diagram for ecologists. J Statistical Software. 2007 Sep;22(4):1-20. doi: 10.18637/jss.v022.i04

28. Dray S. spacemakeR: Spatial modelling. R package version 0.0-5/r113; 2013 [acessado em 03 ago 2020]. Disponível em: https://rdrr.io/rforge/spacemakeR/

29. Wilkinson L. 1. venneuler: Venn and Euler Diagrams. R package version 1.1-0; 2011 [acessado em 03 ago 2020]. Disponível em: http://CRAN.R-project.org/package=venneuler

30. Renka RJ, Eglen S, Zuyev S, White D. tripack: Triangulation of irregularly Spaced Data. Fortran code. $\mathrm{R}$ package version 1.3-6; 2013 [acessado em 10 jul 2015]. Available from: https://cran.rproject.org/web/packages/tripack/index.html

31. Mueller-Dombois D, Ellenberg H. Aims and methods of vegetation ecology. New Jersey (US): The Blackburn Press; 2002.

32. Brower JE, Zar JH. Field and laboratory methods for general ecology. Dubuque (IA): WMC Brow; 1984.

33. Botrel RT, Oliveira Filho AT, Rodrigues LA, Curi N. Composição florística e estrutura da comunidade arbórea de um fragmento de floresta estacional semidecidual em Ingaí, MG, e a influência de variáveis ambientais na distribuição das espécies. Rev Brasil Bot. 2002 Jun;25(2):195-213. doi: 10.1590/S010084042002000200008

34. EMBRAPA. Centro Nacional e Pesquisa em Solos. Sistema brasileiro de classificação de solos. 2. ed. Brasília (DF): Embrapa-SPI; Rio de Janeiro: Embrapa-Solos; 2006.

35. Rossi CQ, Polidoro JC. Ciclos dos nutrientes e sua relação com a nutrição de plantas. In: Tavares SRL, Melo AS, Andrade AG, Rossi CQ, Capeche CL, Balieiro FC, et al. Curso de recuperação de áreas degradadas: a visão da Ciência do Solo no contexto do diagnóstico, manejo, indicadores de monitoramento e estratégias de recuperação. Rio de Janeiro: Embrapa Solos; 2008.

36. Souza RF, Machado AM, Galvão F, Figueiredo Filho A. Effect of environmental variables in the distribution of tree species in the Iguaçu national park. Rev Árvore. 2017 Nov;41(6):1-10. doi: 10.1590/1806-90882017000600009

37. Carvalho WAC, Oliveira Filho AT, Fontes MAL, Curi N. Variação espacial da estrutura da comunidade arbórea de um fragmento de floresta semidecídua em Piedade do Rio Grande, MG, Brasil. Braz J Bot. 2007 Jun;30(2):315-35. doi: 10.1590/S0100-84042007000200015

38. Loebens RS, Silva ACS, Higuchi P, Mafra AL, Silva JO, Gonçalves DA, et al. Partição da variação florístico-estrutural do componente arbóreo em uma floresta ombrófila mista aluvial no sul do Brasil. Ciência Florestal. 2018 Abr-Jun;28(2):554-66. doi: 10.5902/1980509832038

39. Maracahipes-Santos L, Lenzab E, Santos JO, Mewsd HA, Oliveira B. Effects of soil and space on the woody species composition and vegetation structure of three Cerrado phytophysionomies in the Cerrado-Amazon transition. Braz J Biol. 2017 Nov;77(4):830-9. doi: 10.1590/1519-6984.02016

40. Dias PB. Florística e estrutura em ambiente de borda-interior em remanescente de floresta ombrófila densa das terras baixas [dissertação]. Jerônimo Monteiro (ES): Universidade Federal do Espírito Santo; 2019. $120 \mathrm{p}$.

41. Lewis RJ, Pakeman RJ, Mars RH. Identifying the multi-scale spatial structure of plant community determinants of an important national resource. J Vegetation Sci. 2014 Mar;25(1):184-97. doi: 10.1111/jvs. 12071

42. Diniz-Filho JAF, Siqueira T, Padial AA, Rangel TF, Landeiro VL, Bini LM. Spatial autocorrelation analysis allows disentangling the balance between neutral and niche processes in metacommunities. Oikos. 2012 Dez;121(2):201-10. doi: 10.1111/j.1600-0706.2011.19563.x

43. Penã-Claros M, Poorter L, Alarcón A. Soil effects on forest structure and diversity in a moist and a Dry Tropical Forest. Biotropica. 2012 May;44(3):276-83. doi: 10.2307 / 41495998

44. Paschoal EM. Análise florística e estrutural em bordas de um fragmento de Floresta Estacional Semidecidual, município de Capelinha, MG [dissertação]. Diamantina (MG): Universidade Federal dos Vales do Jequitinhonha e Mucuri; 2015. 32 p.

45. Silva LS, Costa TR, Vieira AD, Franco SAP, Machado ELM. Variações na composição florística em floresta estacional semidecidual em Curvelo-MG. Nativa. 2019 Jan/Fev;7(1):109-16. doi: 10.31413/nativa.v7i1.6508

46. Rocha SJSS, Torres CMME, Jacovine LAG, Schettini BLS, Villanova PH, Rufino MPMX, et al. Efeito da borda na estrutura e estoque de carbono de uma Floresta Estacional Semidecidual. Adv For Sci. 2019 Feb;6(2):645-50. doi: 10.34062/afs.v6i2.7635

47. Santos MF, Serafim H, Sano PT. Composição e estrutura arbórea em floresta estacional semidecidual no Espinhaço Meridional (Serra do Cipó, MG). Rodriguésia. 2012 Dec;63(4):985-97. doi: 10.1590/S2175-78602012000400015 
48. Borges KF, Azevedo MAM. Inventário florístico de um remanescente de Floresta Estacional Semidecidual no Sul do Espírito Santo, Brasil: Parque Estadual Cachoeira da Fumaça. Rodriguésia. 2017 Dec;68(5):1963-76. doi: 10.1590/2175-7860201768527

49. Gressler E, Pizo MA, Morellato P. Polinização e dispersão de sementes em Myrtaceae do Brasil. Rev Bras Bot. 2006 Dec;29(4):509-30. doi: 10.1590/S0100-84042006000400002

50. Cantuária PC, Alves CMG, Medeiros TDS, Silva RBL, Freitas JL, Cantuária MF, et al. Ocorrência de Fabaceae da Área de Proteção Ambiental da Fazendinha, Macapá, Amapá, Brasil. Biota Amazônia. 2017 Jun;7(2):49-52. doi: 10.18561/2179-5746/biotaamazonia.v7n2p49-52

51. Caiafa AN, Martins FR. Forms of rarity of tree species in the southern Brazilian Atlantic rainforest. Biodiv Conserv. 2010 Nov;19: 2597-618. doi: 10.1007/s10531-010-9861-6

52. Lima JPC, Leão JRA. Dinâmica de crescimento e distribuição diamétrica de fragmentos de florestas nativa e plantada na Amazônia sul ocidental. Floresta e Ambiente. 2013 Mar;20(1):70-9. doi: 10.4322/floram.2012.065

53. Calixto JTJR, Drumond MA. Estudo comparativo da estrutura fitossociológica de dois fragmentos de Caatinga em níveis diferentes de conservação. Pesq Florestal Bras. 2014 Out-Dez;34(80):345-55. doi: 10.4336/2014.pfb.34.80.670

54. Redin CG, Longhi RV, Watzlawick LF, Longhi SJ. Composição florística e estrutura da regeneração natural do Parque Estadual do Espinilho, RS. Ciênc Rural. 2011 Jul;41(7):1195-201. doi: 10.1590/S0103-84782011005000083 